\title{
Computations with Frobenius powers
}

\author{
Susan M. Hermiller* and Irena Swanson**
}

\begin{abstract}
It is an open question whether tight closure commutes with localization in quotients of a polynomial ring in finitely many variables over a field. Katzman $[\mathrm{K}]$ showed that tight closure of ideals in these rings commutes with localization at one element if for all ideals $I$ and $J$ in a polynomial ring there is a linear upper bound in $q$ on the degree in the least variable of reduced Gröbner bases in reverse lexicographic ordering of the ideals of the form $J+I^{[q]}$. Katzman conjectured that this property would always be satisfied. In this paper we prove several cases of Katzman's conjecture. We also provide an experimental analysis (with proofs) of asymptotic properties of Gröbner bases connected with Katzman's conjectures.
\end{abstract}

\section{Introduction}

Throughout this paper $F$ is a field of positive prime characteristic $p, R$ is a finitely generated polynomial ring $F\left[x_{1}, \ldots, x_{n}\right]$ over $F, J$ and $I$ denote ideals of $R$, and $q=p^{e}$ denotes a power of $p$, where $e$ is a non-negative integer. Then $I^{[q]}$ is the eth Frobenius power of $I$, defined by

$$
I^{[q]}:=\left(i^{q} \mid i \in I\right)
$$

It follows that if $I$ is generated by $f_{1}, \ldots, f_{r}$, then $I^{[q]}$ is generated by $f_{1}^{q}, \ldots, f_{r}^{q}$.

The main motivation for our work in this paper is the theory of tight closure, in which Frobenius powers of ideals play a central role. In particular, we address the question of whether tight closure commutes with localization. The basics of tight closure can be found in the first few sections of $[\mathrm{HH}]$; however, in the following paper no knowledge of tight closure will be needed.

The polynomial ring $R$ is a regular ring, so every ideal in $R$, and in the localizations of $R$, is tightly closed [HH, Theorem 4.4], and hence tight closure commutes with localization in $R$. However, it is not known if tight closure commutes with localization in quotient rings $R / J$ of $R$, even for the special case of localization at a multiplicatively closed set $\left\{1, r, r^{2}, r^{3}, \ldots\right\}$, generated by one element $r \in R / J$. Katzman $[\mathrm{K}]$ showed that for this special case it suffices to consider the case $r=x_{n}$ (by possibly modifying $R, I$, and $J$ ).

\footnotetext{
* The first author acknowledges support from NSF grant DMS-0071037

** The second author acknowledges support from NSF grant DMS-9970566
} 2000 Mathematics Subject Classification. 13P10, 13A35

Key words and phrases. Frobenius powers, Gröbner bases, tight closure, binomial ideals. 
Katzman also proved that a positive answer to the question of tight closure commuting with localization at $x_{n}$ would be provided by a positive answer to the following conjecture.

Conjecture: (Katzman [K, Conjecture 4]) Let $R=F\left[x_{1}, \ldots, x_{n}\right]$ where $F$ is a field of characteristic $p$, and let $I$ and $J$ be ideals of $R$. Let $G_{q}$ be the reduced Gröbner basis for the ideal $J+I^{[q]}$ with respect to the reverse lexicographic ordering. Then there exists an integer $\alpha$ such that the degrees in $x_{n}$ of the elements of $G_{q}$ are bounded above by $\alpha q$.

The (graded) reverse lexicographic ordering on monomials in $x_{1}, \ldots, x_{n}$ is defined by $x_{1}^{a_{1}} x_{2}^{a_{2}} \cdots x_{n}^{a_{n}}<x_{1}^{b_{1}} x_{2}^{b_{2}} \cdots x_{n}^{b_{n}}$ if $\sum_{i} a_{i}<\sum_{i} b_{i}$, or if $\sum_{i} a_{i}=\sum_{i} b_{i}$ and $a_{i}>b_{i}$ for the last index $i$ at which $a_{i}$ and $b_{i}$ differ. For background on reduced Gröbner bases, and Buchberger's algorithm for finding these bases, see for example [CLO].

Katzman's conjecture holds trivially when $J=(0)$, since Frobenius powers commute with sums in rings of characteristic $p$, and hence the reduced reverse lexicographic Gröbner basis for $I^{[q]}$ consists of the $q$ th powers of elements of the reduced Gröbner basis for $I$. The other known cases are due to Katzman, who proved that the conjecture also holds whenever $J$ is generated by monomials [K, Theorem 8], and whenever $J$ is generated by binomials and simultaneously $I$ is generated by monomials [K, Corollary 11]. There are classes of examples for which it is known that tight closure commutes with localization but for which Katzman's conjecture has not been proved; in particular, one such class, due to Smith [S], consists of ideals $I$ and $J$ for which $J$ is a binomial ideal and $I$ is arbitrary. Since the question of whether tight closure commutes with localization has so far defied proof for quotient rings of polynomial rings, accordingly the proof of Katzman's conjecture is expected to be hard. Difficulties in finding a general proof include the dependence of Gröbner bases on the characteristic of the field $F$ and the dependence of Gröbner bases on raising a subset of the generators to powers.

In this paper we study the asymptotic behavior of three functions of $q$ associated to the family of reduced reverse lexicographic Gröbner bases $G_{q}$ for the ideals $J+I^{[q]}$, namely

(1) the maximum of the $x_{n}$-degrees of the elements of $G_{q}$ (as in Katzman's conjecture), also written as the $x_{n}$-degree of $G_{q}$ and denoted $\delta(q)$;

(2) the maximum of the total degrees of the elements of $G_{q}$, also referred to as the total degree of $G_{q}$ and denoted $\Delta(q)$; and

(3) the cardinality $c(q)$ of $G_{q}$.

Since for any ideals $I$ and $J, \delta(q) \leq \Delta(q)$ for all $q$, a linear upper bound for $\Delta(q)$ also implies Katzman's conjecture.

In Section 2 of this paper we prove (Theorem 2.1) that Katzman's conjecture holds for polynomial rings in one or two variables with arbitrary ideals $I$ and $J$, and find a linear upper bound for $\Delta(q)$ and a constant upper bound for $c(q)$ as well. (As part of the proof of this theorem, we include a review of the steps of the Buchberger algorithm for reduced Gröbner basis computation.)

We prove in Theorem 2.5 that Katzman's conjecture holds in the case in which $I$ is principal and $J$ is arbitrary. Moreover, we show that the total degree function $\Delta(q)$ is also bounded above by a linear function. Note that in this case, Hochster and Huneke [HH] showed that tight closure commutes with localization. (In particular, by [HH, Proposition 6.25(a)] it suffices to assume that the ring $R / J$ is an integral domain, whence by $[\mathrm{HH}$, 
Corollary 5.8] for principal ideals tight closure equals integral closure, which commutes with localization.)

In Sections 3 and 4 we provide further information about the specific form of the functions $\delta(q)$ and $\Delta(q)$, as well as the function $c(q)$, in the more restrictive case in which $I$ and $J$ are both principal binomial (and not monomial) ideals, both to gain better understanding of these functions and to find (constructive) proofs of special cases of Katzman's conjecture with potential for application in more general cases. In Section 3 we compute (in Theorem 3.2) Gröbner bases for the ideals $J+I^{[q]}$ for ideals $I=\left(x^{u}\left(x^{v}-g x^{w}\right)\right.$ ) and $J=\left(x^{a}\left(x^{b}-h x^{c}\right)\right)$ whenever $g$ and $h$ are units, $\operatorname{gcd}\left(x^{u}, x^{w}\right)=1=\operatorname{gcd}\left(x^{b}, x^{c}\right)$, and $\left(x^{v}-g x^{w}, x^{b}-h x^{c}\right)=R$, and hence obtain a constructive proof of upper bounds for $\delta(q)$, $\Delta(q)$, and $c(q)$ in this case.

In Theorem 3.3 we prove that for "most" principal binomial ideals $I$ and $J$, there is a change of variables that converts $I$ and $J$ into monoidal ideals, i.e., ideals generated by binomials of the form $u-v$, where $u$ and $v$ are (monic) terms, so that the coefficients are restricted to +1 and -1 . This change of variables preserves both the reverse lexicographic ordering on the monomials and all three of the functions $\delta(q), \Delta(q)$, and $c(q)$. When $I$ and $J$ are monoidal ideals, the quotient rings $R /\left(J+I^{[q]}\right)$ are monoid rings over $F$ for finitely presented commutative monoids, and the Gröbner bases for the ideals $J+I^{[q]}$ can also be considered to be finite complete rewriting systems in the category of commutative monoids.

In Section 4 we study the asymptotic behavior of the three functions $\delta(q), \Delta(q)$, and $c(q)$ for constructions of the reduced Gröbner bases $G_{q}$ for a wide range of examples of principal monoidal ideals $I$ and $J$. We give examples illustrating that the three functions can be linear, periodic, or have linear expressions holding only for $q$ sufficiently large; in addition, we show examples in which the cardinality and the $x_{n}$-degree of the Gröbner bases can be bounded above by a constant for all $q$. We also discuss the dependence of the three functions on the characteristic $p$ of the field $F$ for several of the examples. Section 4 ends with a table summarizing the range of types of behavior of the Gröbner bases we computed. Finally, in the Appendix we include a sample of the Macaulay2 [GS] code we used to generate Gröbner bases for small values of $q$ as an aid to our proofs.

\section{Special cases of Katzman's conjecture}

As mentioned in the introduction, several special cases of Katzman's conjecture are known to be true, in which $J=(0)$, or $J$ is generated by monomials (with arbitrary ideal $I$ ), or $J$ is generated by binomials and $I$ by monomials $[\mathrm{K}]$. In all three cases, Katzman's proof also shows a linear upper bound for the function $\Delta(q)$.

We prove next another special case of Katzman's conjecture, namely for $n \leq 2$.

Theorem 2.1: $\quad$ Katzman's conjecture holds when $R$ is a polynomial ring in one or two variables over $F$. Moreover, for any ideals $I$ and $J$ in $R$ and reduced Gröbner basis $G_{q}$ for the ideal $J+I^{[q]}$ with respect to the reverse lexicographic ordering, there exist integers $\alpha$ and $\beta$ such that the $x_{n}$-degree and total degree functions satisfy $\delta(q) \leq \Delta(q) \leq \alpha q$ and the cardinality function satisfies $c(q) \leq \beta$ for all $q$.

Proof: If $R$ is a polynomial ring in one variable, then $R$ is a principal ideal domain, so 
$I=(f)$ and $J=(g)$ for some $f, g \in R$. In this case $J+I^{[q]}$ is also a principal ideal, and the reduced Gröbner basis of $J+I^{[q]}$ consists of the element $\operatorname{gcd}\left(g, f^{q}\right)$, whose total degree is bounded above by $q \operatorname{deg} f$. Then if we define $\alpha:=\operatorname{deg} f$ and $\beta:=1$, we obtain $\delta(q) \leq \Delta(q) \leq \alpha q$ and $c(q)=\beta$ for all $q$.

Next suppose that $R$ is a polynomial ring in two variables $x$ and $y$ over $F$. By earlier observations, we may assume that $I$ and $J$ are non-zero ideals. Let $S$ be a generating set for the ideal $J$, and $T$ a generating set for $I$; choose $S$ and $T$ so that the leading coefficients of all of their elements are 1. Define $T_{q}:=\left\{t^{q} \mid t \in T\right\}$ to be the corresponding generating set for $I^{[q]}$.

We apply the Buchberger algorithm with the reverse lexicographic ordering to compute a Gröbner basis of $J+I^{[q]}$, starting with the generating set $S \cup T_{q}$. At each step, a partial Gröbner basis $B_{i-1}:=S \cup T_{q} \cup\left\{p_{1}, \ldots, p_{i-1}\right\}$ has been found, and an $S$-polynomial of a pair of elements in $B_{i-1}$ is computed and reduced with respect to all of the elements in this basis. If the result is non-zero, the polynomial is divided by its leading coefficient and the resulting monic polynomial is added as the element $p_{i}$ to form the basis $B_{i}$. When there are no non-zero reduced $S$-polynomials remaining, this creates a Gröbner basis $B:=S \cup T_{q} \cup\left\{p_{1}, \ldots, p_{k}\right\}$ for $J+I^{[q]}$ with respect to the reverse lexicographic ordering, where each element of $B$ is a monic polynomial, and for each $1 \leq i \leq k$, all of the terms of the polynomial $p_{i}$ are reduced with respect to $S \cup T_{q} \cup\left\{p_{1}, \ldots, p_{i-1}\right\}$.

In order to compute the reduced Gröbner basis $G_{q}$ of $J+I^{[q]}$, we need to reduce the Gröbner basis $B$. For each polynomial $r \in B$, replace $r$ in the basis with the monic polynomial obtained by reducing all of the terms of $r$ with respect to the elements of $B \backslash\{r\}$, and dividing by the resulting leading coefficient. Repeat this process for all of the polynomials in the basis, removing any zero polynomials that result, until no more reduction can be done. This gives the reduced Gröbner basis $G_{q}$ for $J+I^{[q]}[$ CLO, Prop. 2.7.6].

The total degree of the reduced Gröbner basis $G_{q}$ for $J+I^{[q]}$ will be at most the total degree for the basis $B$. To compute bounds on these degrees, we first need to describe the polynomials $p_{i}$ more carefully.

Let $x^{a} y^{b}$ be the leading term of a non-zero element $p$ of $S$. In particular, since $J=(S) \neq(0)$, there is a non-zero monic polynomial $p^{\prime} \in J$, and by adding the element $x y p^{\prime} \in J$ to the set $S$ if necessary, we may assume (for ease of notation) that both $a$ and $b$ are non-zero. For each $1 \leq i \leq k$, let $x^{a_{i}} y^{b_{i}}$ be the leading term of the polynomial $p_{i}$ in $B$. Since $p_{i}$ is reduced with respect to $S$, either $0 \leq a_{i}<a$ or $0 \leq b_{i}<b$, or both. If $i>j$, then $p_{i}$ is also reduced with respect to $p_{j}$. More specifically, at each step of the algorithm described above, when $p_{i}$ is computed, (at least) one of four possible cases occurs. Either

(1) $0 \leq a_{i}<a$ and $a_{i} \neq a_{j}$ for all $1 \leq j \leq i-1$,

(2) $0 \leq a_{i}<a$ and for some $j<i, a_{i}=a_{j}$ and $b_{i}<b_{j}$,

(3) $0 \leq b_{i}<b$ and $b_{i} \neq b_{j}$ for all $1 \leq j \leq i-1$, or

(4) $0 \leq b_{i}<b$ and for some $j<i, b_{i}=b_{j}$ and $a_{i}<a_{j}$.

In cases (2) and (4), the total degree of $p_{i}$ is strictly less than the maximal total degree of the previous basis $S \cup T_{q} \cup\left\{p_{1}, \ldots, p_{i-1}\right\}$. In cases (1) and (3), the total degree of the polynomial $p_{i}$, which is a reduction of an $S$-polynomial of a pair of elements in the previous basis, can be at most twice the maximal total degree of the previous basis (by 
definition of S-polynomials). Note that cases (1) and (3) can occur at most $a+b$ times during the algorithm. The maximal total degree of elements in $S \cup T_{q}$ satisfies

$$
\begin{aligned}
\operatorname{deg}\left(S \cup T_{q}\right) & =\max \left\{\operatorname{deg}(S), \operatorname{deg}\left(T_{q}\right)\right\}=\max \{\operatorname{deg}(S), q \cdot \operatorname{deg}(T)\} \\
& \leq q \cdot \max \{\operatorname{deg}(S), \operatorname{deg}(T)\} .
\end{aligned}
$$

Thus the total degree of the basis $B$ is at most $2^{a+b} \cdot q \cdot \max \{\operatorname{deg}(S), \operatorname{deg}(T)\}$. If we define the constant $\alpha:=2^{a+b} \cdot \max \{\operatorname{deg}(S), \operatorname{deg}(T)\}$, then this proves that $\Delta(q) \leq \alpha q$. Since for all $q, \delta(q) \leq \Delta(q) \leq \alpha q$, therefore Katzman's conjecture holds in the case in which the polynomial ring has two variables.

Finally, to get the bound on the cardinality of the reduced Gröbner basis $G_{q}$ of $J+I^{[q]}$, note that although the element $p \in S$ with leading term $x^{a} y^{b}$ may have been reduced or removed in the reduction process to construct $G_{q}$ from $B$, no polynomial that remains in $G_{q}$ may have leading term divisible by $x^{a} y^{b}$. For each number $0 \leq a^{\prime}<a$ and $0 \leq b^{\prime}<b$, there can be at most one polynomial in $G_{q}$ with leading term of the form $x^{a^{\prime}} y^{*}$ for any number $*$, and at most one polynomial in $G_{q}$ with leading term $x^{*} y^{b^{\prime}}$. Then the cardinality of $G_{q}$ satisfies $\left|G_{q}\right| \leq a+b$. Then by defining the constant $\beta:=a+b$, we obtain $c(q) \leq \beta$.

In order to prove Katzman's conjecture in the case in which $I$ is principal and $J$ is arbitrary, we first need to consider the effect of (de)homogenization on Gröbner bases. If $R=F\left[x_{1}, \ldots, x_{n}\right], z$ is a new variable over $R$, and $R^{\prime}=F\left[x_{1}, \ldots, x_{n}, z\right]$, then for any polynomial $f \in R$, let $f^{h}$ denote the homogenization of $f$ in $R^{\prime}$ with respect to $z$. Define the dehomogenization homomorphism $\phi: R^{\prime} \rightarrow R$ by $x_{i} \mapsto x_{i}$ and $z \mapsto 1$. The following lemma is well-known but we could not find it in the literature. We include the proof here for completeness.

Lemma 2.2: $\quad$ Suppose that $K=\left(f_{1}, \ldots, f_{k}\right)$ is an ideal of $R=F\left[x_{1}, \ldots, x_{n}\right], R^{\prime}=$ $F\left[x_{1}, \ldots, x_{n}, z\right]$, and $K^{h}:=\left(\left\{f_{i}^{h} \mid i=1, \ldots, k\right\}\right)$ is a partial homogenization of $K$. Let $G^{\prime}$ be the reduced Gröbner basis for $K^{h}$ with respect to the (graded) reverse lexicographic ordering with z lexicographically least (i.e. $z<x_{i}$ for all $i$ ). Then the set $G:=\left\{\phi(g) \mid g \in G^{\prime}\right\}$ is a Gröbner basis for $K$ with respect to the (graded) reverse lexicographic ordering in $R$.

Proof: For each generator $f_{i}$ of $K, f_{i}^{h}=\sum p_{j} g_{j}$ for some $p_{j} \in R^{\prime}$ and $g_{j} \in G^{\prime}$. Then $f_{i}=\phi\left(f_{i}^{h}\right)=\sum \phi\left(p_{j}\right) \phi\left(g_{j}\right)$, so $G$ is a basis for the ideal $K$.

The ideal $K^{h}$ is homogeneous, so the Gröbner basis $G^{\prime}$ must consist of homogeneous polynomials [CLO, Theorem 8.3.2]. For a homogeneous polynomial $g \in G^{\prime}$, the $z$-degree of the initial (leading) term $i n(g)$ is minimal among all of the terms of $g$. For any non-leading term of $g$ with the same $z$-degree as in $(g)$, the image under $\phi$ of the non-leading term must have the same degree, but be strictly less in the reverse lexicographic ordering, than that of $\phi(i n(g))$. Therefore, $\phi(i n(g))=i n(\phi(g))$ for all $g \in G^{\prime}$, where the ordering on monomials of $R$ is the (graded) reverse lexicographic ordering restricted to the variables $x_{1}, \ldots, x_{n}$. For each $g, h \in G^{\prime}$, the $S$-polynomial $S(g, h)=0$ since $G^{\prime}$ is a Gröbner basis. For the corresponding elements of $G$, the fact that $\phi$ preserves leading terms gives $S(\phi(g), \phi(h))=$ $\phi(S(g, h))=\phi(0)=0$. Therefore $G$ is a Gröbner basis for $K$ with respect to the reverse lexicographic ordering. 
Note that the Gröbner basis $G$ in the lemma above may not be reduced.

The second step leading to the main result of this section on the special case of Katzman's conjecture is an analysis of graded components of monomial ideals. For any ideal $K$, the initial ideal $\operatorname{in}(K)$ is the monomial ideal consisting of the leading terms of the elements of $K$ with respect to the reverse lexicographic ordering. For any monomial ideal $L$ in $R$, there is a uniquely defined minimal generating set for $L$, denoted $M G(L)$, consisting of the monic monomials of $L$ which are not divisible by any other monomial of $L$. Let $\max \operatorname{deg} M G(L)$ denote the maximum of the total degrees of the elements of $M G(L)$. In the case in which $L=i n(K)$, the set $M G(i n(K))$ is the set of leading terms of the reduced reverse lexicographic Gröbner basis for $K$, and $\max \operatorname{deg} M G(i n(K))$ is the maximum of the total degrees of the elements of this basis.

For any homogeneous ideal $K$ in $R$, let $K_{d}$ denote the subspace of degree $d$ homogeneous polynomials in $K$. The Hilbert function of $K$ is the function $H F_{K}: \mathbb{N} \rightarrow \mathbb{N}$ defined by $H F_{K}(d):=\operatorname{dim} R_{d} / K_{d}$, where $\operatorname{dim}$ denotes vector space dimension over $F$. For a monomial ideal $L$, the graded Betti number $\beta_{1 j}(R / L)$ denotes the number of elements of the minimal generating set $M G(L)$ of degree $j$. Let $L_{\geq D}:=\sum_{d \geq D} L_{d}$ be the ideal generated by the union of the subspaces of $L$ of degree at least $D$.

Lemma 2.3: Let $L$ be a monomial ideal of $R=F\left[x_{1}, \ldots, x_{n}\right]$. Then for any $D \in \mathbb{N}, L_{\geq D}$ is a monomial ideal such that

(1) $\left(L_{\geq D}\right)_{d}=\left\{\begin{array}{ll}0 & \text { if } d<D \\ L_{d} & \text { if } d \geq D\end{array}\right.$ and hence $H F_{L_{\geq D}}(d)= \begin{cases}\operatorname{dim} R_{d} & \text { if } d<D \\ H F_{L}(d) & \text { if } d \geq D .\end{cases}$

(2) $\max \operatorname{deg} M G(L) \leq D$ iff $M G\left(L_{\geq D}\right) \subseteq R_{D}$ iff $\beta_{1 j}\left(R / L_{\geq D}\right)=0$ for all $j \neq D$.

Proof: The proof is straightforward from the definitions and from the fact that $M G\left(L_{\geq D}\right)=\left(M G(L) \cap L_{\geq D}\right) \cup$

$\{x y \mid x \in M G(L), \operatorname{deg}(x)<D$, and $y$ is a monic monomial of degree $(D-\operatorname{deg}(x))\}$.

In the third step leading to the proof of Katzman's conjecture when $I$ is principal, we consider the action of graded automorphisms on monomial ideals. The graded automorphisms of $R=F\left[x_{1}, \ldots, x_{n}\right]$ are the elements of $G L(n, F)$. For any $g \in G L(n, F)$ and any monomial ideal $L$, the ideal $g(L)$ is another monomial ideal. The set of all upper triangular matrices in $G L(n, F)$ forms the Borel subgroup. A result of Pardue [P, Proposition 6] says that if a monomial ideal $L$ is invariant under the Borel subgroup action, then whenever $M$ is a monomial in $L, 1 \leq i<j \leq n$, and $l$ is the largest integer such that $x_{j}^{l} \mid M$, then $\left(\frac{x_{i}}{x_{j}}\right)^{l} M \in L$.

The lexicographic ordering on monomials is defined by $x_{1}^{a_{1}} x_{2}^{a_{2}} \cdots x_{n}^{a_{n}}<_{l e x} x_{1}^{b_{1}} x_{2}^{b_{2}} \cdots x_{n}^{b_{n}}$ if $a_{i}<b_{i}$ for the first index $i$ at which $a_{i}$ and $b_{i}$ differ. Let $M=x_{1}^{a_{1}} x_{2}^{a_{2}} \cdots x_{s}^{a_{s}}$ be a monomial with $a_{s}>0$ and $s>1$. The immediate successor of $M$ is the monomial $m=x_{1}^{a_{1}} x_{2}^{a_{2}} \cdots x_{s-1}^{a_{s-1}+1} x_{s}^{a_{s}-1}$; that is, $M<_{\text {lex }} m$ and $\operatorname{deg}(M)=\operatorname{deg}(m)$, and there is no other monomial $m^{\prime}$ with the same degree for which $M<_{\text {lex }} m^{\prime}<_{\text {lex }} m$. A monomial ideal $L$ is said to be lexicographic if whenever $M$ is a monomial in $L$ and $m$ is the monomial in $R$ which is the immediate successor of $M$, then $m$ is also in $L$; it follows that every monomial in $R$ of the same degree as $M$ but greater in the lexicographic ordering will also be in $L$.

Lemma 2.4: Let $L$ be a monomial ideal of $R=F\left[x_{1}, \ldots, x_{n}\right]$ that is invariant under 
the action of the Borel subgroup, and suppose $D \geq \max \operatorname{deg} M G(L)$. Then $L_{\geq n D}$ is a lexicographic ideal.

Proof: Suppose $M$ is a monomial in $L_{\geq n D}$. Then $M \in L$, so $M=M_{0} M_{1}$ for some $M_{0} \in M G(L)$ (hence $\operatorname{deg}\left(M_{0}\right) \leq D$ ) and for some $M_{1} \in R_{u}$ with $u \geq n D-\operatorname{deg}\left(M_{0}\right) \geq$ $(n-1) D$. Write $M_{0}=x_{1}^{l_{1}} \cdots x_{s}^{l_{s}}$ with $l_{s}>0$ and $M_{1}=x_{1}^{r_{1}} \cdots x_{t}^{r_{t}}$ with $r_{t}>0$. Let $m$ be the immediate successor of $M$.

If $t \geq s$, then $M=x_{1}^{l_{1}+r_{1}} \cdots x_{s}^{l_{s}+r_{s}} \cdots x_{t}^{r_{t}}$. Define $m_{1}:=x_{1}^{r_{1}} \cdots x_{t-1}^{r_{t-1}+1} x_{t}^{r_{t}-1}$. In this case, $m=M_{0} m_{1} \in L_{\operatorname{deg}(M)} \subset L_{\geq n D}$.

Suppose that $s>t$. Since $\sum r_{i}=u \geq(n-1) D$, there exists an index $i$ with $1 \leq i \leq t<s \leq n$ such that $r_{i} \geq D$. Since $\operatorname{deg}\left(M_{0}\right) \leq D, l_{s} \leq D \leq r_{i}$ also. Define $m_{0}:=\left(x_{1}^{l_{1}} \cdots x_{s-1}^{l_{s-1}}\right) x_{i}^{l_{s}}$ and $m_{1}:=\left(x_{1}^{r_{1}} \cdots x_{t}^{r_{t}}\right) x_{i}^{-l_{s}} x_{s-1} x_{s}^{l_{s}-1}$. Then $m=m_{0} m_{1}, m_{1} \in R_{u}$, and Pardue's result above [P, Proposition 6] says that $m_{0}=\left(x_{i} / x_{s}\right)^{l_{s}} M_{0} \in L$. Therefore $m \in L_{\geq n D}$ in this case as well.

As the last step leading to the proof of the main theorem of this section, Theorem 2.5, we consider several properties of homogeneous ideals preserved by the action of (all) graded automorphisms in $G L(n, F)$ (where in each case, the proof that the group action preserves the property is straightforward from the definition). For any homogeneous ideal $K$ in $R$ and any $g \in G L(n, F)$, the ideal $g(K)$ is another homogeneous ideal in $R$. The Hilbert functions $H F_{K}(d)=H F_{g(K)}(d)$ are invariant under $g$. The action of $g \in G L(n, F)$ also preserves Krull dimension of the corresponding quotient rings; that is, $\operatorname{dim}(R / K)=\operatorname{dim}(R / g(K))$. The homogeneous ideal $K$ is defined to have regularity $\operatorname{reg}(K)=m$ if the $j$ th syzygy module of $K$ is generated in degrees $\leq m+j$ for all $j \geq 0$, and $m$ is minimal with respect to this property (see [E, Section 20.5] for more details). Regularity is also invariant under the action of a graded automorphism, so $\operatorname{reg}(K)=\operatorname{reg}(g(K))$ for $g \in G L(n, F)$.

Theorem 2.5: Let $I$ and $J$ be ideals in $R=F\left[x_{1}, \ldots, x_{n}\right]$, with $I$ principal. Then Katzman's conjecture holds for $J+I^{[q]}$. Moreover, there exists an integer $\alpha$ such that for all $q$, the total degree of elements of a reduced Gröbner basis of $J+I^{[q]}$ is bounded above by $\alpha q$.

Proof: Gröbner bases do not change after replacing $F$ by its algebraic closure, so without loss of generality we may assume that $F$ is algebraically closed, and hence $F$ is also infinite. Note that it suffices to prove the linear upper bound on the total degree of elements of the Gröbner basis of $J+I^{[q]}$. For ease of notation, let $K:=J+I^{[q]}$.

Write $I=(f)$ and $J=\left(f_{2}, \ldots, f_{k}\right)$. Set $R^{\prime}:=F\left[x_{1}, \ldots, x_{n}, z\right]$, and let $I^{h}=\left(f^{h}\right)$ and $J^{h}=\left(f_{2}^{h}, \ldots, f_{k}^{h}\right)$ be the partial homogenizations with respect to $z$. Then $I^{h}$ is a principal ideal in $R^{\prime}$, and for all $q,\left(I^{h}\right)^{[q]}$ is the homogenization of $I^{[q]}$. Also, $J^{h}+\left(I^{h}\right)^{[q]}=$ $\left(\left(f^{q}\right)^{h}, f_{2}^{h}, \ldots, f_{k}^{h}\right)$ is a partial homogenization of $J+I^{[q]}$. The total degree of a non-reduced Gröbner basis for $J+I^{[q]}$ is at least as large as the total degree of the reduced Gröbner basis for the same ordering, so Lemma 2.2 shows that the total degree of the reduced reverse lexicographic Gröbner basis for $J^{h}+\left(I^{h}\right)^{[q]}$ is an upper bound for the total degree of the reduced Gröbner basis for $J+I^{[q]}$. Thus it suffices to prove upper bounds on $\Delta(q)$ for the Gröbner bases of $J^{h}+\left(I^{h}\right)^{[q]}$. Therefore for the remainder of this proof we may assume that both $I$ and $J$, and hence also $K=J+I^{[q]}$, are homogeneous ideals in $R$. 
Since $I$ is principal, $I^{[q]}$ is a power of $I$. Then by [K, Theorem 21] (or an extension of $[\mathrm{Sw}$, Theorem 3.6]), there exists an integer $\phi$ such that for all $q$, the regularity $\operatorname{reg}(K) \leq$ $\phi q$. A result of Bayer-Stillman [BS2, Proposition 1 and Lemma 5] says that there exists a Zariski-open subset $U$ of $G L(n, F)$, depending on $I, J$, and $q$, such that for all $g \in$ $U$, the monomial ideal $i n(g(K))$ is invariant under the action of the Borel subgroup, and for all $0 \leq i \leq \operatorname{dim}(R / g(K))-1=\operatorname{dim}(R / K)-1, x_{n-i}$ is not a zero divisor on $R /\left(i n(g(K)), x_{n}, \ldots, x_{n-(i-1)}\right)^{\text {sat }}$, where ${ }^{\text {sat }}$ denotes the saturation of the ideal. Then [BS1, Theorem 2.4 and Corollary 2.5] says that $i n(g(K))$ is generated by elements of degree at most the regularity of $g(K)$. Since the field $F$ is infinite, $U$ is not empty. For the remainder of the proof, we fix an element $g \in U$; then $i n(g(K))$ is invariant under the action of the Borel subgroup, and since regularity is invariant under $g$, the maximal total degree of an element of the minimal generating set max $\operatorname{deg} M G(i n(g(K)) \leq \operatorname{reg}(g(K))=\operatorname{reg}(K) \leq \phi q$.

Hilbert functions are preserved both by graded automorphisms and by taking the initial ideal [CLO, Proposition 9.3.9], so $H F_{i n(K)}=H F_{K}=H F_{g(K)}=H F_{\text {in }(g(K))}$. Using Lemma 2.3(1), then the Hilbert functions $H F_{i n(K)_{\geq n \phi q}}=H F_{i n(g(K))_{\geq n \phi q}}$ as well. Lemma 2.4 says that the ideal $i n(g(K))_{\geq n \phi q}$ is a lexicographic ideal. Then by Pardue [P, Theorem 31], the graded Betti numbers satisfy

$$
\beta_{1 j}\left(R / i n(K)_{\geq n \phi q}\right) \leq \beta_{1 j}\left(R / i n(g(K))_{\geq n \phi q}\right) .
$$

Since max $\operatorname{deg} M G\left(i n(g(K)) \leq \phi q \leq n \phi q\right.$, Lemma $2.3(2)$ says that $\beta_{1 j}\left(R / i n(g(K))_{\geq n \phi q}\right)=$ 0 for $j \neq n \phi q$, and so $\beta_{1 j}\left(R / i n(K)_{\geq n \phi q}\right)=0$ for $j \neq n \phi q$ as well. Applying the other direction of Lemma $2.3(2)$, then max $\operatorname{deg} M G(i n(K)) \leq n \phi q$. Therefore the elements of the minimal generating set of the ideal $i n(K)$, and hence also the elements of the reduced reverse lexicographic Gröbner basis for $K=J+I^{[q]}$, have total degree at most $n \phi q$. If we define the constant $\alpha:=n \phi$, then $\delta(q) \leq \Delta(q) \leq \alpha q$.

\section{Principal binomial ideals: General constructions}

For the remainder of the paper we direct our attention to the case in which the ideals $I$ and $J$ are principal and binomial, and obtain more detailed information about the specific form of the degree functions $\delta(q)$ and $\Delta(q)$, as well as the cardinality function $c(q)$. We begin by considering arbitrary monoidal binomials which generate the whole ring.

Lemma 3.1: Let $F$ be a field and let $R=F\left[x_{1}, \ldots, x_{n}\right]$ be a polynomial ring in $n$ variables over $F$. Let $x^{v}-g x^{w}, x^{b}-h x^{c} \in R$, where $v, w, b, c$ are $n$-tuples of non-negative integers, $g, h$ are units in $F, \operatorname{gcd}\left(x^{v}, x^{w}\right)=1=\operatorname{gcd}\left(x^{b}, x^{c}\right)$, and in reverse lexicographic ordering, $x^{v}>x^{w}$ and $x^{b}>x^{c}$. Assume that $\left(x^{v}-g x^{w}, x^{b}-h x^{c}\right)=R$. Then $w=c=\underline{0}$, and there is a positive rational number $l$ such that $v_{i}=l b_{i}$ for all $i$.

Proof: If the conclusion holds after tensoring with the algebraic closure $\bar{F}$ of $F$ over $F$, then it also holds in $R$. So without loss of generality we may assume that $F$ is algebraically closed.

The hypothesis on the ordering implies that $v$ and $b$ are both non-zero. If both $w$ and $c$ are also both non-zero, then $R=\left(x^{v}-g x^{w}, x^{b}-h x^{c}\right) \subseteq\left(x_{1}, \ldots, x_{n}\right) R$, which is a contradiction. So either $w$ or $c$ is zero; without loss of generality suppose that $w=\underline{0}$. 
Choose any $\left(k_{1}, \ldots, k_{n}\right) \in F^{n}$ such that $k^{v}=g$. Choose $i$ such that $v_{i}>0$. Since $v_{i}>0$, then $k_{i} \neq 0$, and $k_{i}$ depends on the choices of the other $k_{j}$ by the relation

$$
k_{i}=g^{1 / v_{i}} \prod_{j \neq i, v_{j} \neq 0} k_{j}^{-v_{j} / v_{i}}
$$

(for some choice of the $v_{i}$ th roots). Since $k^{v}-g k^{w}=g-g=0$, the assumption that $\left(x^{v}-g x^{w}, x^{b}-h x^{c}\right)=R$ implies that $k^{b}-h k^{c}$ is a unit in $F$. In particular, for all indices $j$ with $v_{j}=0$, any choice of $x_{j}=k_{j} \in F$ for these indices makes

$$
k^{b}-h k^{c}=g^{b_{i} / v_{i}} \prod_{j \neq i, v_{j} \neq 0} k_{j}^{b_{j}-b_{i}\left(v_{j} / v_{i}\right)} \prod_{v_{j}=0} x_{j}^{b_{j}}-h g^{c_{i} / v_{i}} \prod_{j \neq i, v_{j} \neq 0} k_{j}^{c_{j}-c_{i}\left(v_{j} / v_{i}\right)} \prod_{v_{j}=0} x_{j}^{c_{j}}
$$

a unit in $F$.

Suppose that $m$ is an index such that $m \neq i$ and $v_{m}=0$. If $b_{m}>0$ and $c_{m}>0$, then for the choice of $x_{m}=0$ the displayed expression above is $k^{b}-h k^{c}=0$, which is not a unit, giving a contradiction. If $b_{m}=0$ and $c_{m} \neq 0$, then with the choice of $k_{j}=1$ and $x_{j}=1$ for all $j \neq i, m$ and $x_{m}=\left(h^{-1} g^{\left(b_{i}-c_{i}\right) / v_{i}}\right)^{1 / c_{m}}$, the expression is again zero and not a unit, giving a contradiction. Similar choices show that the case in which $b_{m} \neq 0$ and $c_{m}=0$ cannot occur. Therefore when $v_{m}=0$, we have that $b_{m}=c_{m}=0$. Thus $b_{m}-c_{m}=0=\left(b_{i}-c_{i}\right)\left(v_{m} / v_{i}\right)$ for all indices $m \neq i$ with $v_{m}=0$.

Next let $m$ be any index such that $m \neq i$ and $v_{m} \neq 0$. If in addition $k_{1}, \ldots, k_{n}$ are all chosen to be non-zero, then

$$
k^{b-c}-h=g^{\left(b_{i}-c_{i}\right) / v_{i}} \prod_{j \neq i} k_{j}^{b_{j}-c_{j}-\left(b_{i}-c_{i}\right)\left(v_{j} / v_{i}\right)}-h
$$

is also a unit in $F$. If $b_{m}-c_{m}-\left(b_{i}-c_{i}\right)\left(v_{m} / v_{i}\right) \neq 0$, then for the choice of $k_{j}=1$ for all $j \neq i, m$, and the choice of $k_{m}=\left[h g^{-\left(b_{i}-c_{i}\right) / v_{i}}\right]^{1 /\left(b_{m}-c_{m}-\left(b_{i}-c_{i}\right)\left(v_{m} / v_{i}\right)\right)}$, the expression above is $k^{b-c}-h=0$, giving a contradiction. So $b_{m}-c_{m}-\left(b_{i}-c_{i}\right)\left(v_{m} / v_{i}\right)=0$, or $b_{m}-c_{m}=\left(b_{i}-c_{i}\right)\left(v_{m} / v_{i}\right)$, when $v_{m} \neq 0$ also.

Thus for all $j \neq i$, we have that $b_{j}-c_{j}=\left(b_{i}-c_{i}\right)\left(v_{j} / v_{i}\right)$ and $v_{j} / v_{i}$ is non-negative. By hypothesis $x^{b}>x^{c}$ in the reverse lexicographic ordering, so we must have $b_{i}-c_{i}>0$ and $b_{j} \geq c_{j}$ for all $j$. By the assumption that $\operatorname{gcd}\left(x^{b}, x^{c}\right)=1$, it follows that $c=\underline{0}$. Then $b_{i} v_{j}=b_{j} v_{i}$ for all $j$, and since $v_{i} \neq 0$ and $b \neq \underline{0}, b_{i} \neq 0$ as well. Therefore if we define the positive rational number $l:=v_{i} / b_{i}$, then $v_{j}=l b_{j}$ for all $j$.

This result leads to the following definition. Two binomials $x^{u}\left(x^{v}-g x^{w}\right)$ and $x^{a}\left(x^{b}-\right.$ $\left.h x^{c}\right)$ with $x^{v}>x^{w}$ and $x^{b}>x^{c}$ are of the same type if there are non-negative integers $l$ and $m$ and $n$-tuples $B$ and $C$ of non-negative integers with $x^{B}>x^{C}$ such that $v=l B$, $w=l C, b=m B$, and $c=m C$; in this case, we say the binomials are of type $(B, C)$. With this notation the Lemma above says that if the ideal generated by two non-monomial binomials is the whole ring, then the two binomials are both of type $(B,(0, \ldots, 0))$ for some $B$, and neither binomial is a multiple of any variable. The corresponding result fails for a 3-generated binomial ideal; for example, the three binomials $x_{1}-1, x_{2}-1, x_{1} x_{2}-2$ generate the whole ring, yet no two of the three binomials are of the same type. 
The following theorem shows that for the ideals considered in Lemma 3.1, Theorem 2.5 has a stronger conclusion, namely one can in addition bound the number of elements in the reduced Gröbner bases, as well as giving constructive upper bounds for the $x_{n}$-degree and total degree.

Theorem 3.2: $\quad$ Let $F$ be a field of positive prime characteristic $p$ and $R=F\left[x_{1}, \ldots, x_{n}\right]$ a polynomial ring in $n$ variables over $F$. Let $I=\left(x^{u}\left(x^{v}-g x^{w}\right)\right)$ and $J=\left(x^{a}\left(x^{b}-h x^{c}\right)\right)$ be ideals in $R$, where $u, v, w, a, b, c$ are $n$-tuples of non-negative integers, $g, h$ are units in $F, \operatorname{gcd}\left(x^{v}, x^{w}\right)=1=\operatorname{gcd}\left(x^{b}, x^{c}\right)$, and in reverse lexicographic ordering, $x^{v}>x^{w}$ and $x^{b}>x^{c}$. Assume that $\left(x^{v}-g x^{w}, x^{b}-h x^{c}\right)=R$. Then for $q$ sufficiently large, the maximal $x_{n}$-degree of the Gröbner basis of $J+I^{[q]}$ is $\delta(q) \leq \max \left(\left(u_{n}+v_{n}\right) q, a_{n}+b_{n}\right)$, the maximal total degree is $\Delta(q) \leq \max ((|u|+|v|) q,|a|+|b|)$, and the cardinality of the Gröbner basis is $c(q) \leq 4$.

Proof: By Lemma 3.1, $w=c=0$ and the generators of $I$ and $J$ have the same type. Then $I^{[q]}=\left(x^{q u}\left(x^{q v}-g^{q}\right)\right)$ and $J=\left(x^{a}\left(x^{b}-h\right)\right)$. We will explicitly compute a Gröbner basis for $J+I^{[q]}$.

The hypothesis that $\left(x^{v}-g, x^{b}-h\right)=R$ implies that there are polynomials $r, s \in R$ with $r\left(x^{v}-g x^{w}\right)+s\left(x^{b}-h x^{c}\right)=1$. Taking $q$ th powers of both sides and then multiplying by $\operatorname{lcm}\left(x^{q u}, x^{a}\right)$ yields

$r^{q} \frac{\operatorname{lcm}\left(x^{q u}, x^{a}\right)}{x^{q u}} x^{q u}\left(x^{q v}-g^{q}\right)+\left[s^{q}\left(x^{b}-h x^{c}\right)^{q-1}\right] \frac{\operatorname{lcm}\left(x^{q u}, x^{a}\right)}{x^{a}} x^{a}\left(x^{b}-h x^{c}\right)=\operatorname{lcm}\left(x^{q u}, x^{a}\right)$.

Thus $J+I^{[q]}$ contains $\operatorname{lcm}\left(x^{q u}, x^{a}\right)$. Computation of the S-polynomials of this monomial with the two generators of $J+I^{[q]}$ shows that

$$
\begin{gathered}
\frac{1}{g^{q}} S\left(x^{q(u+v)}-g^{q} x^{q u}, \operatorname{lcm}\left(x^{q u}, x^{a}\right)\right)=\frac{1}{g^{q}} \frac{\operatorname{lcm}\left(x^{q(u+v)}, x^{a}\right)}{x^{q(u+v)}} g^{q} x^{q u}=\frac{\operatorname{lcm}\left(x^{q(u+v)}, x^{a}\right)}{x^{q v}} \text { and } \\
\frac{1}{h} S\left(x^{a+b}-h x^{a}, \operatorname{lcm}\left(x^{q u}, x^{a}\right)\right)=\frac{1}{h} \frac{\operatorname{lcm}\left(x^{a+b}, x^{q u}\right)}{x^{a+b}} h x^{a}=\frac{\operatorname{lcm}\left(x^{a+b}, x^{q u}\right)}{x^{b}}
\end{gathered}
$$

are also in $J+I^{[q]}$.

Let $E_{j}:=\frac{\operatorname{lcm}\left(x^{a+j b}, x^{q u}\right)}{x^{j b}}$. By the $S$-polynomial calculation above, $E_{1} \in J+I^{[q]}$. If $E_{j} \in J+I^{[q]}$, then so is

$$
\frac{1}{h} S\left(x^{a}\left(x^{b}-h\right), E_{j}\right)=\frac{\operatorname{lcm}\left(x^{a+b}, \frac{\operatorname{lcm}\left(x^{a+j b}, x^{q u}\right)}{x^{j b}}\right)}{x^{b}} .
$$

The exponent of $x_{i}$ in this equals $\max \left(a_{i}+b_{i}, \max \left(a_{i}+j b_{i}, q u_{i}\right)-j b_{i}\right)-b_{i}=\max \left(a_{i}, \max \left(a_{i}-\right.\right.$ $\left.\left.b_{i}, q u_{i}-(j+1) b_{i}\right)\right)=\max \left(a_{i}, q u_{i}-(j+1) b_{i}\right)$, which is the same as the exponent of $x_{i}$ in $E_{j+1}$. Thus the monic S-polynomial above is $\frac{1}{h} S\left(x^{a}\left(x^{b}-h\right), E_{j}\right)=E_{j+1}$. Therefore all the $E_{j}$ are in $J+I^{[q]}$. Note that the exponent $\max \left(a_{i}, q u_{i}-j b_{i}\right)$ of $x_{i}$ in $E_{j}$ is at least as large as the exponent of $x_{i}$ in $E_{j+1}$ for all $i$, so $E_{j}$ is a multiple of $E_{j+1}$ for each $j$. Thus for sufficiently large $j, E_{j}=E_{j+1}=E_{j+2}=\cdots$, and we denote this eventual monomial as $E_{\infty}$. All of the $E_{j}$ are multiples of $E_{\infty}$. 
Define the set

$$
B:=\left\{x^{q u}\left(x^{q v}-g^{q}\right), x^{a}\left(x^{b}-h\right), \frac{\operatorname{lcm}\left(x^{q(u+v)}, x^{a}\right)}{x^{q v}}, E_{\infty}\right\}
$$

then $B$ is a basis of $J+I^{[q]}$. The S-polynomial of the first two elements is $\frac{\operatorname{lcm}\left(x^{q(u+v)}, x^{a+b}\right)}{x^{q v}} g^{q}-$ $\frac{\operatorname{lcm}\left(x^{q(u+v)}, x^{a+b}\right)}{x^{b}} h$, which reduces modulo the third element in $B$ and modulo $E_{1}$ (i.e. modulo $\left.E_{\infty}\right)$ to zero. The S-polynomial of the first and the third elements in $B$ is

$$
S\left(x^{q u}\left(x^{q v}-g^{q}\right), \frac{\operatorname{lcm}\left(x^{q(u+v)}, x^{a}\right)}{x^{q v}}\right)=\frac{\operatorname{lcm}\left(x^{q(u+v)}, \frac{\operatorname{lcm}\left(x^{q(u+v)}, x^{a}\right)}{x^{q v}}\right)}{x^{q v}} g^{q} .
$$

The exponent of $x_{i}$ in this equals

$$
\max \left(q u_{i}+q v_{i}, \max \left(q u_{i}+q v_{i}, a_{i}\right)-q v_{i}\right)-q v_{i}=\max \left(q u_{i}, a_{i}-2 q v_{i}\right)
$$

For sufficiently large $q$, if $v_{i} \neq 0$ then $\max \left(q u_{i}, a_{i}-2 q v_{i}\right)=q u_{i}=\max \left(q u_{i}, a_{i}-q v_{i}\right)$, and if $v_{i}=0$ then $\max \left(q u_{i}, a_{i}-2 q v_{i}\right)=\max \left(q u_{i}, a_{i}\right)=\max \left(q u_{i}, a_{i}-q v_{i}\right)$. Since $\max \left(q u_{i}, a_{i}-q v_{i}\right)$ also equals the exponent of $x_{i}$ in the third element of the basis $B$, this shows that the Spolynomial of the first and the third element of $B$ reduces to 0 . The S-polynomial of the first and the fourth elements in $B$ is

$$
\left.S\left(x^{q u}\left(x^{q v}-g^{q}\right), E_{\infty}\right)\right)=\frac{\operatorname{lcm}\left(x^{q(u+v)}, E_{\infty}\right)}{x^{q v}} g^{q} .
$$

The exponent of $x_{i}$ in this equals, for $j$ sufficiently large,

$$
\max \left(q u_{i}, \max \left(a_{i}-q v_{i}, q u_{i}-j b_{i}-q v_{i}\right)\right)=\max \left(q u_{i}, a_{i}-q v_{i}\right)
$$

which is the same as the exponent of $x_{i}$ in the third element of $B$. Thus the S-polynomial of the first element of $B$ with any other element of $B$ reduces to 0 . The S-polynomial of the second and third elements is the monomial

$$
\frac{\operatorname{lcm}\left(x^{a+b}, \frac{\operatorname{lcm}\left(x^{q(u+v)}, x^{a}\right)}{x^{q v}}\right)}{x^{b}} h,
$$

for which the exponent of $x_{i}$ is $\max \left(a_{i}, \max \left(q u_{i}-b_{i}, a_{i}-q v_{i}-b_{i}\right)\right)=\max \left(a_{i}, q u_{i}-b_{i}\right)$, so that this S-polynomial is a multiple of $E_{1}$ and thus of $E_{\infty}$, and hence reduces to zero. We have previously established that the S-polynomial of the second and the fourth elements reduces to 0 modulo the given basis. The last two elements of the basis $B$ are both monomials, so their S-polynomial is 0 as well. This proves that for $q$ sufficiently large the set $B$ is a Gröbner basis of $J+I^{[q]}$ with respect to the reverse lexicographic ordering.

Although the Gröbner basis $B$ may not be reduced, the reduced reverse lexicographic Gröbner basis $G_{q}$ for $J+I^{[q]}$ will have cardinality and degrees at most those of $B$. Thus 
we can read off upper bounds for the three functions for $q$ sufficiently large, and find that $\delta(q) \leq \max \left(\left(u_{n}+v_{n}\right) q, a_{n}+b_{n}\right), \Delta(q) \leq \max ((|u|+|v|) q,|a|+|b|)$, and $c(q) \leq 4$.

Next we use Lemma 3.1 to show that the principal monoidal ideals cover "most" of the possibilities for principal binomial ideals.

Theorem 3.3: $\quad$ For any principal binomial (non-monomial) ideals $I$ and $J$ which are generated by binomials that are not of the same type, there is a change of variables under which $I$ and $J$ become principal monoidal ideals. Furthermore, this change of variables preserves the reverse lexicographic ordering and the three functions $\delta(q), \Delta(q)$, and $c(q)$.

Proof: Let $F$ be a field of positive prime characteristic $p$ and $R=F\left[x_{1}, \ldots, x_{n}\right]$ a polynomial ring in $n$ variables over $F$. Since Gröbner bases are unchanged if we pass to $\bar{F}\left[x_{1}, \ldots, x_{n}\right]$, where $\bar{F}$ is the algebraic closure of $F$, without loss of generality we may assume that $F$ is algebraically closed.

Let $I$ and $J$ be arbitrary principal binomial (non-monomial) ideals that are not of the same type. We can write $I=\left(x^{u}\left(x^{v}-g x^{w}\right)\right)$ and $J=\left(x^{a}\left(x^{b}-h x^{c}\right)\right)$, where $u, v, w, a, b$, and $c$ are $n$-tuples of non-negative integers, $g$ and $h$ are units in $F, x^{v}>x^{w}$ and $x^{b}>x^{c}$ in the reverse lexicographic ordering, and $\operatorname{gcd}\left(x^{v}, x^{w}\right)=1=\operatorname{gcd}\left(x^{b}, x^{c}\right)$.

Case I. Suppose there exist non-zero elements $k_{1}, \ldots, k_{n}$ in $F$ such that $k^{v}-g k^{w}=$ $0=k^{b}-h k^{c}$. In this case under the variable change $x_{i} \mapsto k_{i} x_{i}$ for all $i$, the reverse lexicographic ordering is preserved, and the generator of the image $\widetilde{I}$ of $I$ under this ring automorphism is $k^{u} x^{u}\left(k^{v} x^{v}-g k^{w} x^{w}\right)$. After dividing through by the non-zero element $k^{u} k^{v}=k^{u} g k^{w}$ of $F$, this generator becomes $x^{u}\left(x^{v}-x^{w}\right)$. A similar computation holds for the generator of the image $\widetilde{J}$ of $J$; hence the generators of $\widetilde{I}$ and $\widetilde{J}$ are monoidal. As this ring automorphism preserves the reverse lexicographic ordering, it maps Gröbner bases to Gröbner bases. Since this change of variables is linear, the functions $\delta(q), \Delta(q)$, and $c(q)$ will also be preserved.

Case II. Suppose that there do not exist non-zero elements $k_{1}, \ldots, k_{n}$ in $F$ such that $k^{v}-g k^{w}=0=k^{b}-h k^{c}$.

Case IIa. Suppose Case II holds and also that $v_{i}+w_{i}>0$ and $b_{i}+c_{i}=0$ for some index $i$. There is another index $j$ for which either $b_{j}>0$ or $c_{j}>0$, but not both, since $x^{b}>x^{c}$ and $\operatorname{gcd}\left(x^{b}, x^{c}\right)=1$. By performing the change of variables $x_{j} \mapsto h^{1 / b_{j}}$ (respectively $x_{j} \mapsto\left(h^{-1}\right)^{1 / c_{j}}$ ) and $x_{m} \mapsto x_{m}$ for all $m \neq j$, the generator $x^{a}\left(x^{b}-h x^{c}\right.$ ) of $J$ is mapped to a scalar multiple of $x^{a}\left(x^{b}-\tilde{h} x^{c}\right)=x^{a}\left(x^{b}-x^{c}\right)$ with unit $\tilde{h}=1$. At the same time, the generator of $I$ changes to a scalar multiple of $x^{u}\left(x^{v}-\tilde{g} x^{w}\right)$ for another unit $\tilde{g}$ in $F$. Since either $v_{i}>0$ or $w_{i}>0$, we can similarly replace $x_{i}$ by an appropriate scalar multiple of itself so that $x^{u}\left(x^{v}-\tilde{g} x^{w}\right)$ is mapped to a scalar multiple of $x^{u}\left(x^{v}-x^{w}\right)$. Since $b_{i}=c_{i}=0$, the unit $\tilde{h}=1$ remains unchanged under this second map. As in Case $\mathrm{I}$, this change of variables preserves the ordering and the three functions associated to the Gröbner bases.

Case IIb. Suppose Case II holds and $v_{i}+w_{i}=0$ and $b_{i}+c_{i}>0$ for some index $i$. An argument similar to Case IIa shows this case as well.

Case IIc. Suppose Case II holds and that for all indices $i, v_{i}+w_{i}>0$ if and only if $b_{i}+c_{i}>0$. Let $T$ be the set of indices $m$ for which $v_{m}>0$, let $U$ be the set of indices $m$ 
for which $w_{m}>0$, and let $S:=T \cup U$. Let

$$
b_{+}:=\left\{\begin{array}{ll}
b_{j} & \text { if } j \in T \\
0 & \text { if } j \notin T
\end{array} \quad \text { and } \quad b_{-}:= \begin{cases}b_{j} & \text { if } j \in U \\
0 & \text { if } j \notin U\end{cases}\right.
$$

and define $c_{+}$and $c_{-}$similarly. Then $b=b_{+}+b_{-}$and $c=c_{+}+c_{-}$.

Define new variables $y_{m}$ over $F$, where $m$ varies over the set $S$. We will denote the restrictions of the tuples $v, w, b_{+}, b_{-}, c_{+}$, and $c_{-}$to tuples in the indices of $S$ by the same notation. Consider the ideal $\left(y^{v+w}-g, y^{b_{+}+c_{-}}-h y^{b_{-}+c_{+}}\right)$in $F\left[y_{m} \mid m \in S\right]$.

Since the non-leading (monic) term of the first generator is 1 , it follows directly that $y^{v+w}>1$ and $\operatorname{gcd}\left(y^{v+w}, 1\right)=1$. For the second generator, the indices $m$ for which $\left(b_{+}\right)_{m}>0$ satisfy that both $\left(b_{-}\right)_{m}=0=\left(c_{+}\right)_{m}$, and similarly for $c_{-}$, so the supports of the two terms are disjoint. Then $\operatorname{gcd}\left(y^{b_{+}+c_{-}}, y^{b_{-}+c_{+}}\right)=1$ and either $y^{b_{+}+c_{-}}>y^{b_{-}+c_{+}}$or $y^{b_{-}+c_{+}}>y^{b_{+}+c_{-}}$.

Suppose that $\underline{\tilde{k}}$ is a tuple with entries in $F$ (and indices in $S$ ) for which $\tilde{k}^{v+w}-g=0=$ $\tilde{k}^{b_{+}+c_{-}}-h \tilde{k}^{b_{-}+c_{+}}$. Since the product of all of the $\tilde{k}_{m}$ divides $\tilde{k}^{v+w}$, the first equation shows that all of the entries of $\underline{\tilde{k}}$ are non-zero. Define the $n$-tuple $\underline{k} \in F^{n}$ by $k_{j}:=\tilde{k}_{j}$ for $j \in T$, $k_{j}:=\tilde{k}_{j}^{-1}$ for $j \in U$, and $k_{j}:=1$ for $j \notin S$. Then $k_{1}, \ldots, k_{n}$ are non-zero elements in $F$ for which $0=k^{w}\left(\tilde{k}^{v+w}-g\right)=k^{w}\left(k^{v-w}-g\right)=k^{v}-g k^{w}$ and $0=k^{b_{-}+c_{-}}\left(\tilde{k}^{b_{+}+c_{-}}-h \tilde{k}^{b_{-}+c_{+}}\right)=$ $k^{b_{-}+c_{-}}\left(k^{b_{+}-c_{-}}-h k^{-b_{-}+c_{+}}\right)=k^{b}-h k^{c}$, contradicting the hypothesis of Case II. Therefore the equations $y^{v+w}-g=0=y^{b_{+}+c_{-}}-h y^{b_{-}+c_{+}}$have no solutions over $F$. Then Hilbert's Nullstellensatz says that $\left(y^{v+w}-g, y^{b_{+}+c_{-}}-h y^{b_{-}+c_{+}}\right)=F\left[y_{m} \mid m \in S\right]$.

Applying Lemma 3.1, we get that either $b_{+}+c_{-}=0$ or $b_{-}+c_{+}=0$, and we can write $y^{b_{+}+c_{-}}-h y^{b_{-}+c_{+}}$as a scalar multiple of $y^{\hat{b}+\hat{c}}-\hat{h}$ where $\hat{b}+\hat{c}$ is either $b_{+}+c_{-}$or $b_{-}+c_{+}$, and $\hat{h}$ is $h$ or $h^{-1}$, respectively. The last conclusion of Lemma 3.1 says there is a positive rational number $l$ such that $v+w=l(\hat{b}+\hat{c})$. If $b_{+}+c_{-}=0$, then $b=b_{-}, c=c_{+}$, and $v+w=l\left(b_{-}+c_{+}\right)$, so $v=l c_{+}$and $w=l b_{-}$, which contradicts the assumption that both $x^{v}>x^{w}$ and $x^{b}>x^{c}$. Therefore $b_{-}+c_{+}=0$, so $b=b_{+}, c=c_{-}, v=l b$, and $w=l c$. Therefore the generator $x^{u}\left(x^{v}-g x^{w}\right)$ of the ideal $I$ is of the same type as the generator $x^{a}\left(x^{b}-h x^{c}\right)$ of $J$. But this contradicts the hypothesis that the generators of $I$ and $J$ are of distinct types, so Case IIc cannot occur.

Motivated by the preceding theorem, for the remainder of the paper we consider the case in which the ideals $I$ and $J$ are principal and monoidal.

\section{Principal monoidal ideals: Examples}

In this section we report on our calculations of reduced reverse lexicographic Gröbner bases, together with the functions $\delta(q), \Delta(q)$, and $c(q)$, for ideals of the form $J+I^{[q]}$, where $I$ and $J$ are fixed principal monoidal ideals and $q$ varies over powers of the characteristic of the base field $F$. In every example, the three functions either are eventually (for $q>>0$ ) linear or constant functions, or else eventually vary periodically between linear or constant functions. For several of the examples, we also explore in more detail the dependence of the three functions on the characteristic $p$ of the field $F$. The examples included in this Section were chosen from among all of our computations to illustrate all of the possible behaviors we observed for the three functions. 
In the process of finding each of the following examples, we used the symbolic computer algebra program Macaulay2 [GS] to generate Gröbner bases for ideals $J+I^{[q]}$ for small values of $q$ (usually three or four values), and studied the patterns in these bases to guide us in proving the structure of the Gröbner bases for all values of $q$. A sample of the Macaulay2 code used in our calculations is provided in the Appendix.

We begin with an example in which the degree functions are linear functions and the cardinality is a constant.

Proposition 4.1: $\quad$ Let $R=\mathbb{Z} / 3 \mathbb{Z}[x, y, z], I=\left(y^{2} z-x^{2}\right), J=\left(y^{3}-x y\right), p=3$, and $q=3^{e}$. Then the Gröbner basis of $J+I^{[q]}$ with respect to the reverse lexicographic ordering (with $x_{1}=x, x_{2}=y$, and $x_{3}=z$, so that $z<y<x$ ) is

$$
\left\{y^{3}-x y, x^{q-1} y^{2} z^{q}-x^{2 q}, x^{2 q} y-x^{q} y z^{q}, x^{3 q+1}-x^{2 q+1} z^{q}\right\} .
$$

Therefore the maximal z-degree of the Gröbner basis elements for $J+I^{[q]}$ is $\delta(q)=q$, the maximal total degree of the elements is $\Delta(q)=3 q+1$, and the number of elements in the Gröbner basis is $c(q)=4$ for all $q$.

Proof: Define $f:=y^{3}-x y$ and $g:=y^{2 q} z^{q}-x^{2 q}$, so that $f$ and $g$ generate $J$ and $I^{[q]}$, respectively. Before computing $S$-polynomials, we reduce $g$ modulo $\left(y^{3}-x y\right)$. Note that for any monomial $x^{a} y^{b} z^{c}$ with $b \geq 3$, the monomial reduces to $x^{a+1} y^{b-2} z^{c}$. Then the normal form of $x^{a} y^{b} z^{c}$ modulo $f$ is $x^{a+k} y^{b-2 k} z^{c}$, where $b-2(k-1) \geq 3$ and $b-2 k<3$; that is, $(b-3) / 2<k \leq(b-1) / 2$. Then to find the normal form for $y^{2 q} z^{q}$, where $b=2 q$, we need $q-\frac{3}{2}<k \leq q-\frac{1}{2}$, so $k=q-1$, and the normal form is $x^{q-1} y^{2} z^{q}$. Therefore the polynomial $g$ reduces to $g^{\prime}:=x^{q-1} y^{2} z^{q}-x^{2 q}$.

The polynomials $f$ and $g^{\prime}$ are a basis for $J+I^{[q]}$. Let $h$ denote their S-polynomial

$$
h:=S\left(f, g^{\prime}\right)=x^{q-1} z^{q} f-y g^{\prime}=-x^{q} y z^{q}+x^{2 q} y .
$$

The $S$-polynomial

$$
\begin{aligned}
S\left(g^{\prime}, h\right) & =x^{q+1} g^{\prime}-y z^{q} h=-x^{3 q+1}+x^{q} y^{2} z^{2 q} \\
& \equiv-x^{3 q+1}+x^{2 q+1} z^{q},
\end{aligned}
$$

where $\equiv$ denotes a reduction using $g^{\prime}$; let $i:=x^{3 q+1}-x^{2 q+1} z^{q}$ denote the monic scalar multiple of this polynomial. All of the remaining $S$-polynomials in the basis $\left\{f, g^{\prime}, h, i\right\}$ reduce to 0 . Therefore the four elements indeed generate a Gröbner basis, and since no element of the basis may be reduced by any other, this Gröbner basis is also reduced. This proves that the maximal $z$-degree is of the elements of the Gröbner basis $\delta(q)=q$, the maximal total degree is $\Delta(q)=3 q+1$, and the cardinality is $c(q)=4$.

Note: Let $R=\mathbb{Z} / p \mathbb{Z}[x, y, z]$, with $x, y, z$ variables over $\mathbb{Z} / p \mathbb{Z}$, where $p$ is any prime and $q$ varies over powers of $p$. Let $I=\left(y^{2} z-x^{2}\right)$ and $J=\left(y^{3}-x y\right)$ be the same ideals as in the example above. In this case, the same sets as in Proposition 4.1 above are the reduced Gröbner bases of the ideals $J+I^{[q]}$ in characteristic $p$ also. Indeed, the proof above applies, since the hypothesis that $p=3$ was never used in the proof.

The number of elements in the Gröbner bases need not remain constant, as we prove next with the ideals $I$ and $J$ from Proposition 4.1, but with their roles switched. 
Proposition 4.2: $\quad$ Let $R=\mathbb{Z} / 3 \mathbb{Z}[x, y, z], I=\left(y^{2} z-x^{2}\right), J=\left(y^{3}-x y\right)$, and $q=3^{e}$. Then the Gröbner basis of $I+J^{[q]}$ (roles of $I$ and $J$ exchanged) with respect to the reverse lexicographic ordering (with $z<y<x$ ) is

$$
\begin{gathered}
\left\{y^{2} z-x^{2}, y^{3 q}-x^{q} y^{q}, x^{2 k} y^{3 q-2 k}-x^{q+2 k} y^{q-2 k} \mid 1 \leq k \leq(q-1) / 2\right\} \\
\cup\left\{x^{q-1+2 j} y^{2 q+1-2 j}-x^{2 q-1} y z^{j} \mid 1 \leq j \leq q\right\} \cup\left\{x^{3 q+1}-x^{2 q+1} z^{q}\right\} .
\end{gathered}
$$

The corresponding functions for these ideals are $\delta(q)=q, \Delta(q)=3 q+1$ and $c(q)=$ $(3 q+5) / 2$ for all $q$.

Proof: Define the polynomials $f:=y^{2} z-x^{2}, g:=y^{3 q}-x^{q} y^{q}, h_{k}:=x^{2 k} y^{3 q-2 k}-x^{q+2 k} y^{q-2 k}$ when $1 \leq k \leq(q-1) / 2, r_{j}:=x^{q-1+2 j} y^{2 q+1-2 j}-x^{2 q-1} y z^{j}$ when $1 \leq j \leq q$, and $s:=x^{3 q+1}-x^{2 q+1} z^{q}$. Since $q=3^{e}, q$ is odd, so $(q-1) / 2$ is an integer for all values of $e$.

Note that if $q=1$, there are no elements of the form $h_{k}$. In this case, the Gröbner basis is already included in the proof of Proposition 4.1.

Next assume that $q>1$. In this example each of the generators of both $I$ and $J^{[q]}$ is in normal form with respect to the other, giving the first two elements $f$ and $g$ of the basis. The $S$-polynomial

$$
S(f, g)=y^{3 q-2} f-z g=-x^{2} y^{3 q-2}+x^{q} y^{q} z \equiv-x^{2} y^{3 q-2}+x^{q+2} y^{q-2}=-h_{1},
$$

where $\equiv$ denotes a reduction using $f$. Repeating this for $1 \leq k \leq(q-3) / 2$, we get

$$
\begin{aligned}
S\left(f, h_{k}\right) & =x^{2 k} y^{3 q-2 k-2} f-z h_{k}=-x^{2 k+2} y^{3 q-2 k-2}+x^{q+2 k} y^{q-2 k} z \\
& \equiv-x^{2(k+1)} y^{3 q-2(k+1)}+x^{q+2(k+1)} y^{q-2(k+1)}=-h_{k+1}
\end{aligned}
$$

where $\equiv$ denotes a reduction of the second term using $f$. Note that in this $S$-polynomial computation, we required that the first $y$-exponent $3 q-2 k-2 \geq 0$, and to do the later reduction by $f$, we needed that $y^{2}$ divides $y^{q-2 k}$. Then $3 q-2 k \geq 2$ and $q-2 k \geq 2$, so the first inequality is redundant, and the second inequality says $k \leq(q-2) / 2$. Since in this Proposition we are assuming that $p=3$, so $q=3^{e}$ is always odd, the largest value that $k$ can actually reach in this $S$-polynomial computation is $(q-3) / 2$. Then the largest value of $k$ for which a basis element $h_{k}$ is produced is $(q-1) / 2$. Thus the entire set of elements $h_{k}$ is generated in the Buchberger algorithm.

The last element generated this way is $h_{(q-1) / 2}=x^{q-1} y^{2 q+1}-x^{2 q-1} y$. Computing the $S$-polynomial of this and $f$ gives $S\left(f, h_{(q-1) / 2}\right)=-r_{1}$. Again computing $S$-polynomials inductively for $1 \leq j \leq q-1$, we get $S\left(f, r_{j}\right)=-r_{j+1}$. The last element generated in this latter step is $r_{q}=x^{3 q-1} y-x^{2 q-1} y z^{q}$.

Finally, the $S$-polynomial $S\left(f, r_{q}\right)$ reduces (using $f$ ) to the polynomial $-s$, resulting in the last element in the list of the basis elements. It is straightforward to check that with these basis elements all remaining $S$-polynomials reduce to 0 , hence the set is a Gröbner basis, and that the Gröbner basis is reduced. The results on the three functions then follow directly.

Note: Let $R=\mathbb{Z} / 2 \mathbb{Z}[x, y, z]$, so that the characteristic is $p=2$, and let $I=\left(y^{2} z-x^{2}\right)$ and $J=\left(y^{3}-x y\right)$ be the same ideals as in the example above. In the proof above, in the 
computation of the $S$-polynomials $S\left(f, h_{k}\right)$, we noted that the number of polynomials of the form $h_{k}$ produced satisfies $k \leq(q-2) / 2$. When the characteristic $p$ is even, then, the Gröbner basis computation can differ from the proof above at that point. In fact, a proof very similar to the one above shows that for $p=2$ the reduced Gröbner basis of $I+J^{[q]}$ is

$$
\left\{y^{2} z-x^{2}, x^{2 k} y^{3 q-2 k}-x^{q+2 k} y^{q-2 k}, x^{q+2 j} y^{2 q-2 j}-x^{2 q} z^{j} \mid 0 \leq k \leq(q-2) / 2,0 \leq j \leq q\right\}
$$

when $q>1$. Then the functions $\Delta(q)=3 q$ and $c(q)=\frac{3}{2} q+2$ for $q>1$ associated to these Gröbner bases differ from the functions $\Delta(q)$ and $c(q)$ computed in Proposition 4.2 with $p=3$. Thus, not surprisingly, the reduced Gröbner bases do depend on the characteristic of the underlying field in general. In this example, though, the $x_{n}$-degree $\delta(q)=q$ is the same function in both characteristics.

In part (a) of the next Proposition, we show that the function $\delta(q)$ also can equal a constant. In the Propositions above, the functions $\delta(q)$ and $\Delta(q)$ are exactly equal to linear functions, and $c(q)$ equals either a linear or constant function, for all $q$. As mentioned earlier, these functions are not always this regular. Part (b) of the next Proposition illustrates functions $\Delta(q)$ and $c(q)$ which are polynomials eventually but not at the start.

Proposition 4.3: $\quad$ Let $R=\mathbb{Z} / 2 \mathbb{Z}[x, y, z], I=\left(x^{2}-y^{2}\right), J=\left(x y-z^{2}\right)$ and $q=2^{e}$. With the reverse lexicographic ordering (with $z<y<x$ ),

(a) the reduced Gröbner basis for $J+I^{[q]}$ is

$$
\left\{x y-z^{2}, x^{2 q}-y^{2 q}, y^{2 q+1}-x^{2 q-1} z^{2}\right\},
$$

so that $\delta(q)=2, \Delta(q)=2 q+1$ and $c(q)=3$ for all $q$, and

(b) the reduced Gröbner basis for $I+J^{[q]}$ with $q \geq 2$ is

$$
\left\{x^{2}-y^{2}, y^{2 q}-z^{2 q}\right\}
$$

so that in this case $\delta(q)=2 q$,

$$
\Delta(q)=\left\{\begin{array}{ll}
3 & \text { if } q=1, \\
2 q & \text { if } q \geq 2
\end{array} \quad \text { and } \quad c(q)= \begin{cases}3 & \text { if } q=1 \\
2 & \text { if } q \geq 2\end{cases}\right.
$$

for all $q$.

Proof: In the first part, the S-polynomial of the generators $x y-z^{2}$ and $x^{2 q}-y^{2 q}$ of $J$ and $I^{[q]}$, respectively, is $y^{2 q+1}-x^{2 q-1} z^{2}$. The S-polynomials of these three polynomials all reduce to zero. This verifies part (a). In part (b), when $q \geq 2$ the generator $x^{q} y^{q}-z^{2 q}$ of $J^{[q]}$ reduces modulo $I$ to $y^{2 q}-z^{2 q}$. Since the leading terms $x^{2}$ and $y^{2 q}$ of $x^{2}-y^{2}$ and $y^{2 q}-z^{2 q}$ have no common factors, their S-polynomial is zero.

The next example shows that the function $\delta(q)$ may also be a function that is eventually linear but not for small $q$.

Proposition 4.4: $\quad$ Let $R=\mathbb{Z} / 3 \mathbb{Z}[x, y, z, w], I=\left(x^{5} y^{2} z w-x y^{3} z^{2} w\right), J=\left(x y^{2} z^{3} w^{2}-\right.$ $x^{3} y z w^{3}$ ) and $q=3^{e}$. Then with the reverse lexicographic ordering (with $w<z<y<x$ ), the reduced Gröbner basis of $J+I$ is

$$
\left\{x y^{2} z^{3} w^{2}-x^{3} y z w^{3}, x^{5} y^{2} z w-x y^{3} z^{2} w, x^{7} y z w^{3}-x^{3} y^{2} z^{2} w^{3}\right\}
$$

and for $q \geq 3$, the reduced Gröbner basis of $J+I^{[q]}$ is

$$
\left\{x y^{2} z^{3} w^{2}-x^{3} y z w^{3}, x^{6 q-1+2 i} y^{\frac{3 q+1}{2}-i} z w^{\frac{3 q-1}{2}+i}-x^{3 q-2+2 i} y^{2 q+1-i} z^{2} w^{2 q-1+i} \mid 0 \leq i \leq \frac{3 q-1}{2}\right\} \text {. }
$$


Thus

$$
\delta(q)=\left\{\begin{array}{ll}
3 & \text { if } q=1, \\
\frac{7 q-3}{2} & \text { if } q \geq 3,
\end{array} \quad \Delta(q)= \begin{cases}12 & \text { if } q=1 \\
12 q-1 & \text { if } q \geq 3\end{cases}\right.
$$

and $c(q)=\frac{3 q+3}{2}$ for all $q$.

Proof: The case $q=1$ can be computed directly by Macaulay2 and is left to the reader.

Now assume that $q \geq 3$. Define $f:=x y^{2} z^{3} w^{2}-x^{3} y z w^{3}, i_{0}:=\frac{3 q-1}{2}$, and

$$
g_{i}:=x^{6 q-1+2 i} y^{\frac{3 q+1}{2}-i} z w^{\frac{3 q-1}{2}+i}-x^{3 q-2+2 i} y^{2 q+1-i} z^{2} w^{2 q-1+i}
$$

for $0 \leq i \leq i_{0}$. In particular, $g_{i_{0}}=x^{9 q-2} y z w^{3 q-2}-x^{6 q-3} y^{\frac{q+3}{2}} z^{2} w^{\frac{7 q-3}{2}}$.

Observe that whenever $a \geq 1, b \geq 2, c \geq 3$ and $d \geq 2$, the monomial $x^{a} y^{b} z^{c} w^{d}$ reduces to $x^{a+2} y^{b-1} z^{c-2} w^{d+1}$ modulo $J$, so that the normal form of the monomial $x^{a} y^{b} z^{c} w^{d}$ modulo $J$ is $x^{a+2 k} y^{b-k} z^{c-2 k} w^{d+k}$, where $k$ is the largest integer such that $b-(k-1) \geq 2$ and $c-2(k-1) \geq 3$, i.e., $b \geq k+1$ and $c \geq 2 k+1$. In particular, as $q \geq 3$, the generator $x^{5 q} y^{2 q} z^{q} w^{q}-x^{q} y^{3 q} z^{2 q} w^{q}$ of $I^{[q]}$ reduces to $g_{0}=x^{6 q-1} y^{\frac{3 q+1}{2}} z w^{\frac{3 q-1}{2}}-x^{3 q-2} y^{2 q+1} z^{2} w^{2 q-1}$.

Computing the $S$-polynomials, we begin with $S\left(f, g_{i}\right)$ for $i<i_{0}$.

$$
\begin{aligned}
S\left(f, g_{i}\right) & =x^{6 q-2+2 i} y^{\frac{3 q-3}{2}-i} w^{\frac{3 q-5}{2}+i} f-z^{2} g_{i} \\
& =-x^{6 q-1+2(i+1)} y^{\frac{3 q+1}{2}-(i+1)} z w^{\frac{3 q-1}{2}+(i+1)}+x^{3 q-2+2 i} y^{2 q+1-i} z^{4} w^{2 q-1+i} \\
& \equiv-x^{6 q-1+2(i+1)} y^{\frac{3 q+1}{2}-(i+1)} z w^{\frac{3 q-1}{2}+(i+1)}+x^{3 q-2+2(i+1)} y^{2 q+1-(i+1)} z^{2} w^{2 q-1+(i+1)} \\
& =-g_{i+1},
\end{aligned}
$$

where $\equiv$ denotes reduction by $f$. Then for each $i<i_{0}$, the polynomial $g_{i+1}$ must be added to the basis. Computing the remaining $S$-polynomials,

$$
\begin{aligned}
S\left(f, g_{i_{0}}\right) & =x^{9 q-3} w^{3 q-3} f-y z^{2} g_{i_{0}} \\
& =-x^{9 q} y z w^{3 q}+x^{6 q-3} y^{\frac{q+5}{2}} z^{4} w^{\frac{7 q-3}{2}} \\
& \equiv-x^{6 q-1} y^{\frac{q+3}{2}} z^{2} w^{\frac{7 q-1}{2}}+x^{6 q-1} y^{\frac{q+3}{2}} z^{2} w^{\frac{7 q-1}{2}}=0
\end{aligned}
$$

where $\equiv$ denotes reduction by $f$ and $g_{i_{0}}$, and for $i<j$,

$$
\begin{aligned}
S\left(g_{i}, g_{j}\right) & =x^{2(j-i)} w^{j-i} g_{i}-y^{j-i} g_{j} \\
& =-x^{3 q-2+2 j} y^{2 q+1-i} z^{2} w^{2 q-1+j}+x^{3 q-2+2 j} y^{2 q+1-i} z^{2} w^{2 q-1+j}=0 .
\end{aligned}
$$

Therefore $\left\{f, g_{0}, \ldots, g_{i_{0}}\right\}$ is a Gröbner basis of $J+I^{[q]}$; this basis is also reduced. Thus when $q \geq 3$, the maximal $w$-degree of the Gröbner basis is $\delta(q)=\frac{7 q-3}{2}$, the maximal total degree is $\Delta(q)=12 q-1$, and the number of elements is $c(q)=\frac{3 q+3}{2}$.

In the following example we again use the ideals $I$ and $J$ from the previous Proposition and exchange their roles, in order to exhibit periodic behavior of both the cardinality function $c(q)$ and the total degree function $\Delta(q)$ of the elements of the reduced Gröbner 
basis of $I+J^{[q]}$, with periodic behavior starting not with $q=1$ but at the next level, at $q=p$.

Proposition 4.5: $\quad$ Let $R=\mathbb{Z} / 3 \mathbb{Z}[x, y, z, w], I=\left(x^{5} y^{2} z w-x y^{3} z^{2} w\right), J=\left(x y^{2} z^{3} w^{2}-\right.$ $x^{3} y z w^{3}$ ) and $q=3^{e}$. Using the reverse lexicographic ordering (with $w<z<x<y$ ) the reduced Gröbner basis of $I+J$ is

$$
\left\{x^{5} y^{2} z w-x y^{3} z^{2} w, x y^{2} z^{3} w^{2}-x^{3} y z w^{3}, x^{7} y z w^{3}-x^{3} y^{2} z^{2} w^{3}\right\}
$$

the reduced Gröbner basis of $I+J^{[q]}$ for $q$ a positive even power of 3 is

$$
\left\{x^{5} y^{2} z w-x y^{3} z^{2} w, x y^{\frac{9}{4} q-\frac{1}{4}} z^{\frac{13}{4} q-\frac{1}{4}} w^{2 q}-x^{3} y^{\frac{7}{4} q-\frac{3}{4}} z^{\frac{7}{4} q-\frac{3}{4}} w^{3 q}\right\}
$$

and the reduced Gröbner basis of $I+J^{[q]}$ for $q$ an odd power of 3 is

$$
\begin{array}{r}
\left\{x^{5} y^{2} z w-x y^{3} z^{2} w, x^{3} y^{\frac{9}{4} q-\frac{3}{4}} z^{\frac{13}{4} q-\frac{3}{4}} w^{2 q}-x y^{\frac{7}{4} q-\frac{1}{4}} z^{\frac{7}{4} q-\frac{1}{4}} w^{3 q}\right. \\
\left.x y^{\frac{9}{4} q+\frac{1}{4}} z^{\frac{13}{4} q+\frac{1}{4}} w^{2 q}-x^{3} y^{\frac{7}{4} q-\frac{1}{4}} z^{\frac{7}{4} q-\frac{1}{4}} w^{3 q}\right\}
\end{array}
$$

The corresponding functions are given by $\delta(q)=3 q$,

$\Delta(q)=\left\{\begin{array}{ll}12 & \text { if } q=1, \\ \frac{15}{2} q+\frac{3}{2} & \text { if } q=3^{e}, \text { e odd }, \\ \frac{15}{2} q+\frac{1}{2} & \text { if } q=3^{e}, e>0 \text { even }\end{array} \quad\right.$ and $\quad c(q)= \begin{cases}3 \quad \text { if } q=1 \text { or } q=3^{e}, \text { e odd }, \\ 2 \quad \text { if } q=3^{e}, e>0 \text { even }\end{cases}$

for all $q$.

Proof: As in the proof of Proposition 4.4, the case $q=1$ can be computed directly by Macaulay2 and is left to the reader.

Assume that $q \geq 3$; write $q=3^{e}$, with $e>0$. Observe that whenever $a \geq 5, b \geq 2$, $c \geq 1$ and $d \geq 1$, the monomial $x^{a} y^{b} z^{c} w^{d}$ reduces to $x^{a-4} y^{b+1} z^{c+1} w^{d}$ modulo $I$, so the normal form of $x^{a} y^{b} z^{c} w^{d}$ modulo $I$ is $x^{a-4 k} y^{b+k} z^{c+k} w^{d}$, where $k$ is the largest integer such that $a-4(k-1) \geq 5$, i.e., $a \geq 4 k+1$. In reducing the generator $x^{q} y^{2 q} z^{3 q} w^{2 q}-x^{3 q} y^{q} z^{q} w^{3 q}$ of $J^{[q]}$ using the generator $g=x^{5} y^{2} z w-x y^{3} z^{2} w$ of $I$, we need to consider the cases when $e$ is even and odd separately. If $e$ is even, then $q \equiv 1$ ( modulo 4 ), so the monomial $x^{q} y^{2 q} z^{3 q} w^{2 q}$ can be reduced $k=\frac{q-1}{4}$ times and the monomial $x^{3 q} y^{q} z^{q} w^{3 q}$ can be reduced $k=\frac{3 q-3}{4}$ times using $g$. Similarly, if $e$ is odd, then $q \equiv 3$ ( modulo 4), so the monomial $x^{q} y^{2 q} z^{3 q} w^{2 q}$ can be reduced $k=\frac{q-3}{4}$ times and the monomial $x^{3 q} y^{q} z^{q} w^{3 q}$ can be reduced $k=\frac{3 q-1}{4}$ times using $g$. In particular, when $q \geq 3$, the generator $x^{q} y^{2 q} z^{3 q} w^{2 q}-x^{3 q} y^{q} z^{q} w^{3 q}$ of $J^{[q]}$ reduces to $f^{\prime}$, where

$$
f^{\prime}:= \begin{cases}x y^{\frac{9}{4} q-\frac{1}{4}} z^{\frac{13}{4} q-\frac{1}{4}} w^{2 q}-x^{3} y^{\frac{7}{4} q-\frac{3}{4}} z^{\frac{7}{4} q-\frac{3}{4}} w^{3 q}, & e \text { even positive } \\ x^{3} y^{\frac{9}{4} q-\frac{3}{4}} z^{\frac{13}{4} q-\frac{3}{4}} w^{2 q}-x y^{\frac{7}{4} q-\frac{1}{4}} z^{\frac{7}{4} q-\frac{1}{4}} w^{3 q}, & e \text { odd. }\end{cases}
$$

If $e>0$ is even, the S-polynomial of $f^{\prime}$ and the generator $g$ of $I$ is

$$
\begin{aligned}
S\left(g, f^{\prime}\right) & =y^{\frac{9}{4} q-\frac{9}{4}} z^{\frac{13}{4} q-\frac{5}{4}} w^{2 q-1} g-x^{4} f^{\prime} \\
& =-x y^{\frac{9}{4} q+\frac{3}{4}} z^{\frac{13}{4} q+\frac{3}{4}} w^{2 q}+x^{7} y^{\frac{7}{4} q-\frac{3}{4}} z^{\frac{7}{4} q-\frac{3}{4}} w^{3 q} \\
& \equiv-x^{3} y^{\frac{7}{4} q+\frac{1}{4}} z^{\frac{7}{4} q+\frac{1}{4}} w^{3 q}+x^{3} y^{\frac{7}{4} q+\frac{1}{4}} z^{\frac{7}{4} q+\frac{1}{4}} w^{3 q}=0,
\end{aligned}
$$

where $\equiv$ denotes one reduction using $f^{\prime}$ on the first term, and one reduction using $g$ on the second term. For the case in which $e$ is positive and even, this proves that the basis in 
the Proposition is a Gröbner basis; this basis is reduced, and the three functions can be computed directly.

Finally assume that $q$ is an odd power of $p=3$. Then the S-polynomial of $f^{\prime}$ and the generator $g$ of $I$ is

$$
S\left(g, f^{\prime}\right)=y^{\frac{9}{4} q-\frac{11}{4}} z^{\frac{13}{4} q-\frac{7}{4}} w^{2 q-1} g-x^{2} f^{\prime}=-x y^{\frac{9}{4} q+\frac{1}{4}} z^{\frac{13}{4} q+\frac{1}{4}} w^{2 q}+x^{3} y^{\frac{7}{4} q-\frac{1}{4}} z^{\frac{7}{4} q-\frac{1}{4}} w^{3 q},
$$

which is non-zero and reduced with respect to the set $\left\{f^{\prime}, g\right\}$. So let

$$
h:=x y^{\frac{9}{4} q+\frac{1}{4}} z^{\frac{13}{4} q+\frac{1}{4}} w^{2 q}-x^{3} y^{\frac{7}{4} q-\frac{1}{4}} z^{\frac{7}{4} q-\frac{1}{4}} w^{3 q}
$$

be added to $f^{\prime}$ and $g$ in the procedure to form a Gröbner basis of $I+J^{[q]}$. Computing the remaining $S$-polynomials, we find that

$$
\begin{aligned}
S(g, h) & =y^{\frac{9}{4} q-\frac{7}{4}} z^{\frac{13}{4} q-\frac{3}{4}} w^{2 q-1} g-x^{4} h=-x y^{\frac{9}{4} q+\frac{5}{4}} z^{\frac{13}{4} q+\frac{5}{4}} w^{2 q}+x^{7} y^{\frac{7}{4} q-\frac{1}{4}} z^{\frac{7}{4} q-\frac{1}{4}} w^{3 q} \\
& \equiv-x^{3} y^{\frac{7}{4} q+\frac{3}{4}} z^{\frac{7}{4} q+\frac{3}{4}} w^{3 q}+x^{3} y^{\frac{7}{4} q+\frac{3}{4}} z^{\frac{7}{4} q+\frac{3}{4}} w^{3 q}=0
\end{aligned}
$$

where $\equiv$ denotes one reduction using $h$ on the first term and one reduction using $g$ on the second term, and the last $S$-polynomial

$$
\begin{aligned}
S\left(f^{\prime}, h\right) & =y z f^{\prime}-x^{2} h=-x y^{\frac{7}{4} q+\frac{3}{4}} z^{\frac{7}{4} q+\frac{3}{4}} w^{3 q}+x^{5} y^{\frac{7}{4} q-\frac{1}{4}} z^{\frac{7}{4} q-\frac{1}{4}} w^{3 q} \\
& \equiv-x y^{\frac{7}{4} q+\frac{3}{4}} z^{\frac{7}{4} q+\frac{3}{4}} w^{3 q}+x y^{\frac{7}{4} q+\frac{3}{4}} z^{\frac{7}{4} q+\frac{3}{4}} w^{3 q}=0
\end{aligned}
$$

where $\equiv$ denotes one reduction using $g$ on the second term. Therefore $\left\{f^{\prime}, g, h\right\}$ is indeed a Gröbner basis of $I+J^{[q]}$ for $q$ an odd power of $p$. This basis is also reduced, so in this case the maximal $w$-degree of the Gröbner basis is $\delta(q)=3 q$, the maximal total degree is $\Delta(q)=\frac{15 q+3}{2}$, and the number of elements is $c(q)=3$.

In the next example we show that the function $\delta(q)$ also can vary periodically. In the example in Proposition 4.5, $c(q)$ alternated between constant functions for the ideals $J+I^{[q]}$. The next example shows that the function $c(q)$ can vary periodically between linear functions as well. Moreover, the asymptotic patterns for all three functions of the ideals $J+I^{[q]}$ begin further along, at $q=p^{2}$.

Proposition 4.6: Let $R=\mathbb{Z} / 3 \mathbb{Z}[x, y, z], I=\left(x^{2} y^{2} z-x y z^{2}\right), J=\left(x y^{2} z^{5}-x^{2} y z\right)$ and $q=3^{e}$. Then with the reverse lexicographic ordering (with $z<y<x$ ) the reduced Gröbner basis for $J+I$ is

$$
\left\{x y^{2} z^{5}-x^{2} y z, x^{2} y^{2} z-x y z^{2}, x y z^{6}-x^{3} y z\right\}
$$

the reduced Gröbner basis for $J+I^{[3]}$ is

$$
\left\{x y^{2} z^{5}-x^{2} y z, x^{6} y^{6} z^{3}-x^{4} y^{2} z^{2}, x^{7} y^{5} z-x^{4} y^{2} z^{4}, x^{8} y^{4} z-x^{5} y z^{4}, x^{5} y z^{8}-x^{9} y^{3} z\right\}
$$

if $e \geq 2$ is even the reduced Gröbner basis for $J+I^{[q]}$ is

$$
\begin{aligned}
& \left\{x y^{2} z^{5}-x^{2} y z, x^{\frac{9}{4} q-\frac{1}{4}+k} y^{\frac{7}{4} q+\frac{1}{4}-k} z-x^{\frac{3}{2} q-\frac{1}{2}+k} y^{\frac{1}{2} q+\frac{1}{2}-k} z^{2}\right. \\
& \quad x^{\frac{11}{4} q+\frac{1}{4}+j} y^{\frac{5}{4} q-\frac{1}{4}-j} z-x^{2 q-1} y z^{6+4 j}, x^{2 q-1} y z^{2 q+4}-x^{\frac{13}{4} q-\frac{1}{4}} y^{\frac{3}{4} q+\frac{1}{4}} z \\
& \quad \mid 0 \leq k \leq(q-1) / 2,0 \leq j \leq(q-3) / 2\}
\end{aligned}
$$


and if $e \geq 3$ is odd then the reduced Gröbner basis for $J+I^{[q]}$ is

$$
\begin{aligned}
& \left\{x y^{2} z^{5}-x^{2} y z, x^{\frac{9}{4} q-\frac{3}{4}} y^{\frac{7}{4} q+\frac{3}{4}} z^{3}-x^{\frac{3}{2} q-\frac{1}{2}} y^{\frac{1}{2} q+\frac{1}{2}} z^{2}, x^{\frac{9}{4} q+\frac{1}{4}+k} y^{\frac{7}{4} q-\frac{1}{4}-k} z-x^{\frac{3}{2} q-\frac{1}{2}+k} y^{\frac{1}{2} q+\frac{1}{2}-k} z^{4},\right. \\
& x^{\frac{11}{4} q+\frac{3}{4}+j} y^{\frac{5}{4} q-\frac{3}{4}-j} z-x^{2 q-1} y z^{8+4 j}, x^{2 q-1} y z^{2 q+6}-x^{\frac{13}{4} q+\frac{1}{4}} y^{\frac{3}{4} q-\frac{1}{4}} z \\
& \quad \mid 0 \leq k \leq(q-1) / 2,0 \leq j \leq(q-3) / 2\} .
\end{aligned}
$$

The associated functions are

$$
\delta(q)=\left\{\begin{array}{ll}
8 & \text { if } q=3, \\
2 q+4 & \text { if } q=3^{e}, e \geq 0 \text { even, } \\
2 q+6 & \text { if } q=3^{e}, e \geq 3 \text { odd },
\end{array} \quad \Delta(q)= \begin{cases}15 & \text { if } q=3, \\
4 q+4 & \text { if } q=3^{e}, e \geq 0 \text { even, } \\
4 q+6 & \text { if } q=3^{e}, e \geq 3 \text { odd }\end{cases}\right.
$$

$$
\text { and } \quad c(q)= \begin{cases}5 & \text { if } q=3, \\ q+2 & \text { if } q=3^{e}, e \geq 0 \text { even, } \\ q+3 & \text { if } q=3^{e}, e \geq 3 \text { odd }\end{cases}
$$

for all $q$.

Proof: The Gröbner bases for $J+I$ and $J+I^{[3]}$ can be computed with Macaulay2, and are left to the reader. For the rest of the proof, assume $q=p^{e}$ with $e \geq 2$. Let $g=x y^{2} z^{5}-x^{2} y z$ be the generator of the ideal $J$. We need to reduce the generator $x^{2 q} y^{2 q} z^{q}-x^{q} y^{q} z^{2 q}$ of $I^{[q]}$ to normal form modulo $g$. Observe that whenever $a \geq 1, b \geq 2$, and $c \geq 5$, then $x^{a} y^{b} z^{c}$ reduces to $x^{a+1} y^{b-1} z^{c-4}$, so the normal form of the monomial $x^{a} y^{b} z^{c}$ is the monomial $x^{a+k} y^{b-k} z^{c-4 k}$, where $k$ is the largest integer such that $b-(k-1) \geq 2$ and $c-4(k-1) \geq 5$; i.e., $b \geq k+1$ and $c \geq 4 k+1$. For the monomial $x^{2 q} y^{2 q} z^{q}, k$ is the largest integer such that $2 q \geq k+1$ and $q \geq 4 k+1$; in this case, if the latter inequality holds, then the former is true as well, so we only need to find the largest integer $k$ for which $q \geq 4 k+1$. If $e$ is even, then $q \equiv 1$ modulo 4 , so $k=(q-1) / 4$, and the normal form of $x^{2 q} y^{2 q} z^{q}$ is $x^{\frac{9}{4} q-\frac{1}{4}} y^{\frac{7}{4} q+\frac{1}{4}} z$. If $e$ is odd, then $q \equiv 3$ modulo 4 , so $k=(q-3) / 4$, and the normal form of $x^{2 q} y^{2 q} z^{q}$ is $x^{\frac{9}{4} q-\frac{3}{4}} y^{\frac{7}{4} q+\frac{3}{4}} z^{3}$. Similarly, $x^{q} y^{q} z^{2 q}$ reduces $k$ times using $g$ to its normal form when $k$ is the largest integer such that $q \geq k+1$ and $2 q \geq 4 k+1$. As before we can ignore the first inequality. For all $e \geq 2$, we get $k=(2 q-2) / 4=(q-1) / 2$, so the normal form of $x^{q} y^{q} z^{2 q}$ is $x^{\frac{3}{2} q-\frac{1}{2}} y^{\frac{1}{2} q+\frac{1}{2}} z^{2}$. The the normal form for the generator $x^{2 q} y^{2 q} z^{q}-x^{q} y^{q} z^{2 q}$ of $I^{[q]}$ is

$$
f^{\prime}:= \begin{cases}x^{\frac{9}{4} q-\frac{1}{4}} y^{\frac{7}{4} q+\frac{1}{4}} z-x^{\frac{3}{2} q-\frac{1}{2}} y^{\frac{1}{2} q+\frac{1}{2}} z^{2}, & e \text { even } \\ x^{\frac{9}{4} q-\frac{3}{4}} y^{\frac{7}{4} q+\frac{3}{4}} z^{3}-x^{\frac{3}{2} q-\frac{1}{2}} y^{\frac{1}{2} q+\frac{1}{2}} z^{2}, & e \text { odd. }\end{cases}
$$

Suppose that $e \geq 2$ is even. Define the polynomials

$$
\begin{aligned}
f_{k} & :=x^{\frac{9}{4} q-\frac{1}{4}+k} y^{\frac{7}{4} q+\frac{1}{4}-k} z-x^{\frac{3}{2} q-\frac{1}{2}+k} y^{\frac{1}{2} q+\frac{1}{2}-k} z^{2} \quad \text { for } 0 \leq k \leq(q-1) / 2, \\
h_{j} & :=x^{\frac{11}{4} q+\frac{1}{4}+j} y^{\frac{5}{4} q-\frac{1}{4}-j} z-x^{2 q-1} y z^{6+4 j} \quad \text { for } 0 \leq j \leq(q-3) / 2, \text { and } \\
r & :=x^{2 q-1} y z^{2 q+4}-x^{\frac{13}{4} q-\frac{1}{4}} y^{\frac{3}{4} q+\frac{1}{4}} z .
\end{aligned}
$$

Note that $f^{\prime}=f_{0}$. When $0 \leq k \leq(q-3) / 2$, the $S$-polynomial

$$
\begin{aligned}
S\left(g, f_{k}\right) & =x^{\frac{9}{4} q-\frac{5}{4}+k} y^{\frac{7}{4} q-\frac{7}{4}-k} g-z^{4} f_{k} \\
& =-x^{\frac{9}{4} q-\frac{1}{4}+(k+1)} y^{\frac{7}{4} q+\frac{1}{4}-(k+1)} z+x^{\frac{3}{2} q-\frac{1}{2}+k} y^{\frac{1}{2} q+\frac{1}{2}-k} z^{6} \\
& \equiv-x^{\frac{9}{4} q-\frac{1}{4}+(k+1)} y^{\frac{7}{4} q+\frac{1}{4}-(k+1)} z+x^{\frac{3}{2} q-\frac{1}{2}+(k+1)} y^{\frac{1}{2} q+\frac{1}{2}-(k+1)} z^{2}=-f_{k+1},
\end{aligned}
$$


where $\equiv$ denotes a reduction using $g$ on the second term. Therefore the polynomials $f_{k}$ for $0 \leq k \leq(q-1) / 2$ are included with $g$ and $f^{\prime}$ in the procedure to compute the Gröbner basis. The last polynomial in this family is $f_{(q-1) / 2}=x^{\frac{11}{4} q-\frac{3}{4}} y^{\frac{5}{4} q+\frac{3}{4}} z-x^{2 q-1} y z^{2}$. Then

$$
\begin{aligned}
S\left(g, f_{(q-1) / 2}\right) & =x^{\frac{11}{4} q-\frac{7}{4}} y^{\frac{5}{4} q-\frac{5}{4}} g-z^{4} f_{(q-1) / 2} \\
& =-x^{\frac{11}{4} q+\frac{1}{4}} y^{\frac{5}{4} q-\frac{1}{4}} z+x^{2 q-1} y z^{6}=-h_{0} .
\end{aligned}
$$

Similarly, the $S$-polynomial $S\left(g, h_{j}\right)=-h_{j+1}$ for all $0 \leq j \leq(q-5) / 2$, so the polynomials $h_{j}$ for $0 \leq j \leq(q-3) / 2$ are appended to the basis. The final polynomial in this list is $h_{(q-3) / 2}=x^{\frac{13}{4} q-\frac{5}{4}} y^{\frac{3}{4} q+\frac{5}{4}} z-x^{2 q-1} y z^{2 q}$. Then

$$
S\left(g, h_{(q-3) / 2}\right)=x^{\frac{13}{4} q-\frac{9}{4}} y^{\frac{3}{4} q-\frac{3}{4}} g-z^{4} h_{(q-3) / 2}=-x^{\frac{13}{4} q-\frac{1}{4}} y^{\frac{3}{4} q+\frac{1}{4}} z+x^{2 q-1} y z^{2 q+4}=r .
$$

Therefore $r$ is also added to the basis by the Buchberger algorithm. All of the remaining $S$-polynomials reduce to zero modulo this set of polynomials, so the set $\left\{g, f_{k}, h_{j}, r \mid 0 \leq\right.$ $k \leq(q-1) / 2,0 \leq j \leq(q-3) / 2\}$ is a Gröbner basis for $J+I^{[q]}$ in the case that $e \geq 2$ is even.

Finally, suppose that $e \geq 3$ is odd. We have already shown that the polynomials $g=x y^{2} z^{5}-x^{2} y z$ and $f^{\prime}=x^{\frac{9}{4} q-\frac{3}{4}} y^{\frac{7}{4} q+\frac{3}{4}} z^{3}-x^{\frac{3}{2} q-\frac{1}{2}} y^{\frac{1}{2} q+\frac{1}{2}} z^{2}$ are a basis for $J+I^{[q]}$. Define the polynomials

$$
\begin{aligned}
s_{k} & :=x^{\frac{9}{4} q+\frac{1}{4}+k} y^{\frac{7}{4} q-\frac{1}{4}-k} z-x^{\frac{3}{2} q-\frac{1}{2}+k} y^{\frac{1}{2} q+\frac{1}{2}-k} z^{4} \quad \text { for } 0 \leq k \leq(q-1) / 2, \\
t_{j} & :=x^{\frac{11}{4} q+\frac{3}{4}+j} y^{\frac{5}{4} q-\frac{3}{4}-j} z-x^{2 q-1} y z^{8+4 j} \quad \text { for } 0 \leq j \leq(q-3) / 2, \text { and } \\
u & :=x^{2 q-1} y z^{2 q+6}-x^{\frac{13}{4} q+\frac{1}{4}} y^{\frac{3}{4} q-\frac{1}{4}} z .
\end{aligned}
$$

By an argument very similar to the proof above, we get that $S\left(g, f^{\prime}\right)=-s_{0}$ and $S\left(g, s_{k}\right) \equiv$ $-s_{k+1}$ for all $0 \leq k \leq(q-3) / 2$, where $\equiv$ denotes a reduction by $g$. Then $S\left(g, s_{(q-1) / 2}\right)=t_{0}$, and $S\left(g, t_{j}\right)=-t_{j+1}$ when $0 \leq j \leq(q-5) / 2$. Taking one further $S$-polynomial with $g$, $S\left(g, t_{(q-3) / 2}\right)=u$. Finally, all of the remaining $S$ polynomials reduce to 0 modulo these polynomials, so the set $\left\{g, f^{\prime}, s_{k}, t_{j}, u \mid 0 \leq k \leq(q-1) / 2,0 \leq j \leq(q-3) / 2\right\}$ is a Gröbner basis for $J+I^{[q]}$ when $e \geq 3$ is odd.

Since in each case the Gröbner basis we computed is also reduced, the results on the functions associated to these ideals then follow immediately from these bases.

If we change the characteristic in Proposition 4.6 to $p=2$, we find that the $x_{n}$-degree function $\delta(q)$ is dependent on the characteristic of the field $F$ as well; in fact, all three functions $\delta(q), \Delta(q)$ and $c(q)$ are altered, and the periodicity is lost.

Proposition 4.7: Let $R=\mathbb{Z} / 2 \mathbb{Z}[x, y, z], I=\left(x^{2} y^{2} z-x y z^{2}\right), J=\left(x y^{2} z^{5}-x^{2} y z\right)$ and $q=2^{e}$. Then with the reverse lexicographic ordering (with $z<y<x$ ) the reduced Gröbner basis for $J+I^{[q]}$ with $e \geq 3$ is

$$
\begin{aligned}
& \left\{x y^{2} z^{5}-x^{2} y z, x^{\frac{9}{4} q-1} y^{\frac{7}{4} q+1} z^{4}-x^{\frac{3}{2} q-1} y^{\frac{1}{2} q+1} z^{4}, x^{\frac{9}{4} q+j} y^{\frac{7}{4} q-j} z-x^{\frac{3}{2} q+j} y^{\frac{1}{2} q-j} z,\right. \\
& x^{\frac{11}{4} q+k} y^{\frac{5}{4} q-k} z-x^{2 q-1} y z^{5+4 k}, x^{2 q-1} y z^{2 q+5}-x^{\frac{13}{4} q-1} y^{\frac{3}{4} q+1} z^{5} \\
& \mid 0 \leq j \leq(q-2) / 2,0 \leq k \leq(q-2) / 2\} .
\end{aligned}
$$


The associated functions satisfy $\delta(q)=2 q+5, \Delta(q)=4 q+5$ and $c(q)=q+3$ for $q \geq 2^{3}$.

Proof: Assume that $q=2^{e}$ with $e \geq 3$. The main difference between the proof of this Proposition and that of Proposition 4.6 lies in the reduction of the generator $x^{2 q} y^{2 q} z^{q}-$ $x^{q} y^{q} z^{2 q}$ of $I^{[q]}$ modulo the generator $g=x y^{2} z^{5}-x^{2} y z$ of $J$. As in the earlier proof, if $a \geq 1, b \geq 2$, and $c \geq 5$, then the normal form of the monomial $x^{a} y^{b} z^{c}$ is the monomial $x^{a+k} y^{b-k} z^{c-4 k}$, where $k$ is the largest integer such that $b \geq k+1$ and $c \geq 4 k+1$. For the monomial $x^{2 q} y^{2 q} z^{q}$, we get that $k$ is the largest integer such that $q \geq 4 k+1$, i.e. $k \leq(q-1) / 4$. Since $q$ is even, and moreover divisible by 4 , this means that $k=(q-4) / 4$. Then the normal form of $x^{2 q} y^{2 q} z^{q}$ is $x^{\frac{9}{4} q-1} y^{\frac{7}{4} q+1} z^{4}$. Similarly, to find the normal form of $x^{q} y^{q} z^{2 q}$ we reduce the monomial $k$ times where $k$ is the largest integer satisfying $2 q \geq 4 k+1$, or $k \leq(2 q-1) / 4$. Then $k=(2 q-4) / 4$. So the generator of $I^{[q]}$ reduces to the polynomial $f^{\prime}:=x^{\frac{9}{4} q-1} y^{\frac{7}{4} q+1} z^{4}-x^{\frac{3}{2} q-1} y^{\frac{1}{2} q+1} z^{4}$ modulo $g$.

Next define the polynomials $f_{j}:=x^{\frac{9}{4} q+j} y^{\frac{7}{4}} q-j z-x^{\frac{3}{2} q+j} y^{\frac{1}{2} q-j} z$ for $0 \leq j \leq(q-2) / 2$, $h_{k}:=x^{\frac{11}{4} q+k} y^{\frac{5}{4} q-k} z-x^{2 q-1} y z^{5+4 k}$ for $0 \leq k \leq(q-2) / 2$, and $r=: x^{2 q-1} y z^{2 q+5}-$ $x^{\frac{13}{4} q-1} y^{\frac{3}{4} q+1} z^{5}$. Following steps very similar to those in the proof of Proposition 4.6, we find that $S\left(g, f^{\prime}\right) \equiv-f_{0}$ and $S\left(g, f_{j}\right) \equiv-f_{j+1}$ for $0 \leq j \leq(q-4) / 2$, where $\equiv$ denotes a single reduction by $g$ in each case. Also, $S\left(g, f_{(q-2) / 2}\right)=-h_{0}, S\left(g, h_{k}\right)=-h_{k+1}$ for $0 \leq k \leq(q-4) / 2$, and $S\left(g, h_{(q-2) / 2}\right)=r$. All other $S$-polynomials reduce to zero with this basis, and no element of this set can be reduced by any other, so the set $\left\{g, f^{\prime}, f_{j}, h_{k}, r \mid 0 \leq\right.$ $j \leq(q-2) / 2,0 \leq k \leq(q-2) / 2\}$ is a reduced Gröbner basis.

In the final example we show that it need not be the case that the total degree of the Gröbner basis of $J+I^{[q]}$ is bounded above by $q \cdot \max \{\operatorname{Gbdeg} I$, Gbdeg $J\}$, where Gbdeg denotes the total degree of the reduced Gröbner basis (with the reverse lexicographic ordering).

Proposition 4.8: $\quad$ Let $R=\mathbb{Z} / 3 \mathbb{Z}[x, y, z, w]$. The ideal $J+I^{[q]}$ with $I=\left(x^{2} y^{2} z w^{5}-\right.$ $\left.x y z^{2} w^{2}\right), J=\left(x y^{2} z^{3} w-x y z w^{3}\right)$ and $q=3^{e}$ has the reduced Gröbner basis

$$
\left\{x y^{2} z^{3} w-x y z w^{3}, x^{2 q} y^{(3 q+1-2 k) / 2} z w^{6 q-1+2 k}-x^{q} y z^{2 k+2} w^{4 q-2} \mid 0 \leq k \leq(3 q-1) / 2\right\}
$$

with respect to the reverse lexicographic ordering with $w<z<y<x$. Therefore the maximal $w$-degree of the Gröbner basis is $\delta(q)=9 q-2$, the maximal total degree is $\Delta(q)=11 q$, and the number of elements is $c(q)=3(q+1) / 2$ for all $q$.

Therefore $q \cdot \max \{\operatorname{Gbdeg} I, \operatorname{Gbdeg} J\}=q \cdot \max \{10,7\}<11 q=\operatorname{Gbdeg}\left(J+I^{[q]}\right)$.

Proof: Define $f:=x y^{2} z^{3} w-x y z w^{3}$ and for $0 \leq k \leq(3 q-1) / 2$, define

$$
g_{k}:=x^{2 q} y^{(3 q+1-2 k) / 2} z w^{6 q-1+2 k}-x^{q} y z^{2 k+2} w^{4 q-2} .
$$

Note that in this example the generator $x^{2 q} y^{2 q} z^{q} w^{5 q}-x^{q} y^{q} z^{2 q} w^{2 q}$ of $I^{[q]}$ is not in normal form modulo $J$. The term $x^{2 q} y^{2 q} z^{q} w^{5 q}$ reduces (after $(q-1) / 2$ reductions) to $x^{2 q} y^{(3 q+1) / 2} z w^{6 q-1}$, and $x^{q} y^{q} z^{2 q} w^{2 q}$ reduces (using $q-1$ reductions) to $x^{q} y z^{2} w^{4 q-2}$, resulting in the basis element $g_{0}=x^{2 q} y^{(3 q+1) / 2} z w^{6 q-1}-x^{q} y z^{2} w^{4 q-2}$. The $S$-polynomial $S\left(f, g_{0}\right)$ is

$$
S\left(f, g_{0}\right)=-x^{2 q} y^{(3 q+1-2) / 2} z w^{6 q-1+2}+x^{q} y z^{2+2} w^{4 q-2}=-g_{1}
$$


Repeating this, when $k<(3 q-1) / 2$, the $S$-polynomial $S\left(f, g_{k}\right)$ equals $-g_{k+1}$. The remaining $S$-polynomials $S\left(f, g_{(3 q-1) / 2}\right)$ and $S\left(g_{j}, g_{k}\right)$ all reduce to 0 . Thus the polynomials $f$ and $g_{k}$ for $0 \leq k \leq(3 q-1) / 2$ form a Gröbner basis. This Gröbner basis is reduced, giving the results on the functions.

\section{Summary table}

\begin{tabular}{|l|l|l|l|}
\hline Example & $\delta(q)$ & $\Delta(q)$ & $c(q)$ \\
\hline Prop. 4.1, & linear & linear & constant \\
\hline Prop. 4.2 & linear & linear & linear \\
\hline Prop. 4.3(a) & constant & linear & constant \\
\hline Prop. 4.3(b) & linear & linear $(q \geq p)$ & constant $(q \geq p)$ \\
\hline Prop. 4.4 & linear $(q \geq p)$ & linear $(q \geq p)$ & linear \\
\hline Prop. 4.5 & linear & periodically & periodically \\
& linear $(q \geq p)$ & constant $(q \geq p)$ \\
\hline Prop. 4.6 & $\begin{array}{l}\text { periodically } \\
\text { linear }\left(q \geq p^{2}\right)\end{array}$ & $\begin{array}{c}\text { periodically } \\
\text { linear }\left(q \geq p^{2}\right)\end{array}$ & $\begin{array}{c}\text { periodically } \\
\text { linear }\left(q \geq p^{2}\right)\end{array}$ \\
\hline Prop. 4.8 & linear & linear (high coeff. $)$ & linear \\
\hline
\end{tabular}

All of these examples satisfy Katzman's conjecture that the $x_{n}$-degree $\delta(q)$ of the reduced Gröbner basis of $J+I^{[q]}$ is bounded above linearly in $q$. Furthermore, in all of these examples the total degree and cardinality of the Gröbner basis are also bounded above linearly in $q$. However, we are left with the open question of whether the behavior of the functions $\delta(q), \Delta(q)$ and $c(q)$ (eventually) follows one of the patterns in the table above, and whether linear upper bounds on $\delta(q), \Delta(q)$ and $c(q)$ hold, for all ideals $I$ and $J$ in a polynomial ring.

\section{Appendix: Macaulay2 code}

We used variations of the following Macaulay2 code for our calculations, included for the readers interested in making further computations.

Input: polynomial ring $R$, ideals $I, J$

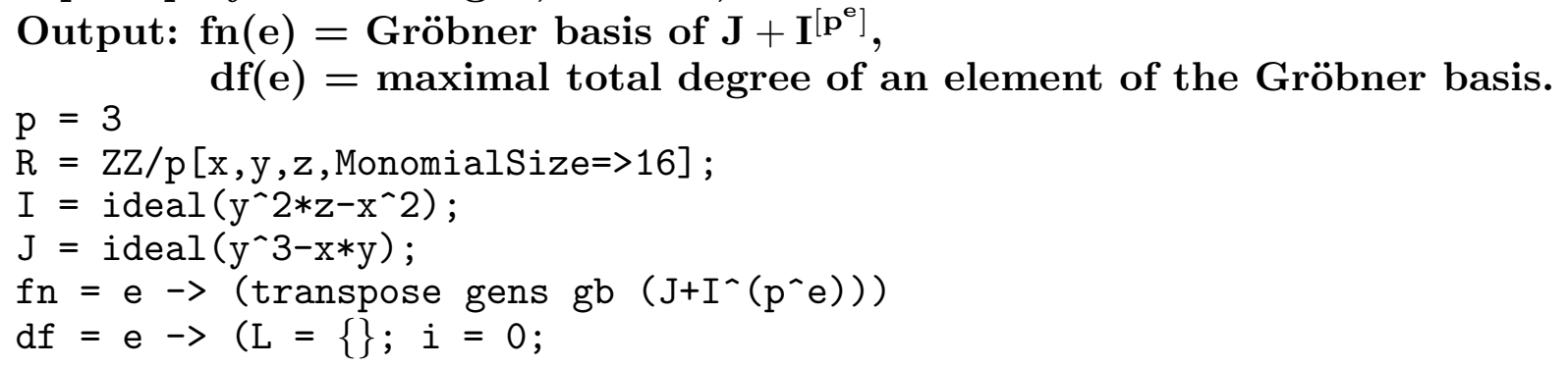




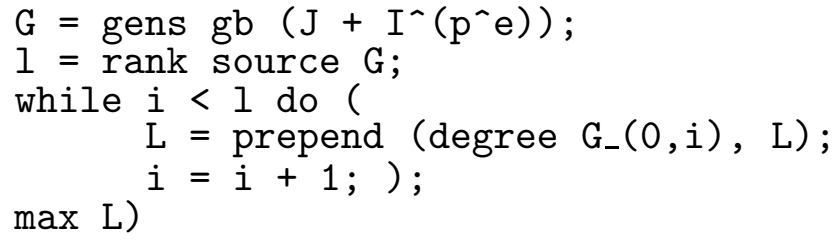

\section{References}

[BS1] D. Bayer and M. Stillman, A criterion for detecting m-regularity, Invent. Math. 87 (1987), 1-11.

[BS2] D. Bayer and M. Stillman, A theorem on refining division orders by the reverse lexicographic order, Duke J. Math. 55 (1987), 321-328.

[CLO] D. Cox, J. Little and D. O'Shea, Ideals, Varieties, and Algorithms: An introduction to computational algebraic geometry and commutative algebra, Undergraduate Texts in Mathematics, Springer-Verlag, New York, 1992.

[E] D. Eisenbud, Commutative Algebra with a View toward Algebraic Geometry, Graduate Texts in Mathematics 150, Springer-Verlag, New York, 1995.

[GS] D. R. Grayson and M. E. Stillman, Macaulay2, a software system for research in algebraic geometry, available at http://www.math.uiuc.edu/Macaulay2.

[HH] M. Hochster and C. Huneke, Tight closure, invariant theory, and the Briançon-Skoda Theorem, J. Amer. Math. Soc. 3 (1990), 31-116.

[K] M. Katzman, The complexity of Frobenius powers of ideals, J. Algebra 203 (1998), 211-225.

[P] K. Pardue, Deformation classes of graded modules and maximal Betti numbers, Illinois J. Math 40 (1996), 564-585.

[S] K. E. Smith, Tight closure commutes with localization in binomial rings, Proc. Amer. Math. Soc. 129 (2001), 667-669.

[Sw] I. Swanson, Powers of ideals: Primary decompositions, Artin-Rees lemma and regularity, Math. Ann. 307 (1997), 299-313.

Department of Mathematics and Statistics, University of Nebraska, Lincoln, Nebraska 68588-0323, USA, E-mail: smh@math.unl.edu

Department of Mathematical Sciences, New Mexico State University, Las Cruces, New Mexico 88003-8001, USA, E-mail: iswanson@nmsu.edu 